\title{
VECTOR-VALUED IMPLICIT LAGRANGIAN FOR SYMMETRIC CONE COMPLEMENTARITY PROBLEMS
}

\author{
Lingchen Kong * \\ Department of Applied Mathematics, Beijing Jiaotong University, \\ Beijing 100044, P. R. China \\ Department of Combinatorics and Optimization, Faculty of Mathematics, University of Waterloo, \\ Waterloo, Ontario N2L 3G1, Canada \\ konglchen@126.com \\ Levent Tunçel \\ Department of Combinatorics and Optimization, Faculty of Mathematics, University of Waterloo, \\ Waterloo, Ontario N2L 3G1, Canada \\ ltuncel@math.uwaterloo.ca \\ Naihua Xiu \\ Department of Applied Mathematics, Beijing Jiaotong University, \\ Beijing 100044, P. R. China \\ nhxiu@bjtu.edu.cn \\ CORR 2006-24 December 18, 2006 (Revised: October 29, 2007; April 3, 2008)
}

\begin{abstract}
The implicit Lagrangian was first proposed by Mangasarian and Solodov as a smooth merit function for the nonnegative orthant complementarity problem. It has attracted much attention in the past ten years because of its utility in reformulating complementarity problems as unconstrained minimization problems. In this paper, exploiting the Jordan-algebraic structure, we extend it to the vector-valued implicit Lagrangian for symmetric cone complementary problem (SCCP), and show that it is a continuously differentiable complementarity function for SCCP and whose Jacobian is strongly semismooth. As an application, we develop the real-valued implicit Lagrangian and the corresponding smooth merit function for SCCP, and give a necessary and sufficient condition for the stationary point of the merit function to be a solution of SCCP. Finally, we show that this merit function can provide a global error bound for SCCP with the uniform Cartesian P-property.
\end{abstract}

Keywords: Symmetric cone complementary problem, Jordan algebra, Vector-valued implicit Lagrangian, C-function, Uniform Cartesian P-property.

AMS Subject Classification: 26B05, 65K05, 90C33

Abbreviated Title: Vector-Valued Implicit Lagrangian for SCCP

\footnotetext{
${ }^{*}$ Corresponding author.
} 


\section{Introduction}

Let $\mathcal{J}$ be a Euclidean Jordan algebra with the inner product $\langle\cdot, \cdot\rangle, K$ be the symmetric cone in $\mathcal{J}$, and $F: \mathcal{J} \rightarrow \mathcal{J}$ be a continuous function. The symmetric cone complementarity problem (SCCP for short) is to find a vector $x \in \mathcal{J}$ such that

$$
x \in K, y \in K,\langle x, y\rangle=0, \quad y=F(x) .
$$

This model provides a simple, natural, and unified framework for various existing complementarity problems, such as the nonnegative orthant nonlinear complementarity problem (NCP), the second-order cone complementarity problem (SOCCP), the semi-definite complementarity problem (SDCP). It has wide applications in engineering, economics, management science, and other fields; we refer the readers to the excellent monographs (Facchinei and Pang 2003, Isac 2000, Luo, Pang and Ralph 1996). Although there exist only several papers addressing the SCCP up-to date (Gowda, Sznajder and Tao 2004, Gowda and Sznajder 2006, Gowda and Tao 2007, Kong and Xiu 2007, Lin and Yoshise 2005, Liu, Zhang and Wang 2006, Malik and Mohan 2003, Malik and Mohan 2006, Sun and Sun 2008, Tao and Gowda 2005, Yoshise 2006), there is a growing trend in the study of SCCP. This paper is mainly concerned with the extension of the implicit Lagrangian for NCP to the setting of SCCP.

The implicit Lagrangian was first proposed by Mangasarian and Solodov (1993) as a smooth merit function for the nonlinear complementarity problem over nonnegative orthant, which is to find a vector $x \in R^{n}$ such that

$$
x \in R_{+}^{n}, y \in R_{+}^{n},\langle x, y\rangle=0, \quad y=F(x) .
$$

It has the following form:

$$
M_{\alpha}(x)=\sum_{i=1}^{n} \phi_{M}\left(x_{i}, F_{i}(x)\right)
$$

with $\phi_{M}: R^{2} \rightarrow R$ being defined by

$$
\phi_{M}(a, b)=a b+\frac{1}{2 \alpha}\left\{\left[(a-\alpha b)_{+}\right]^{2}-a^{2}+\left[(b-\alpha a)_{+}\right]^{2}-b^{2}\right\},
$$

where $\alpha>1$ is any fixed parameter and $(\cdot)_{+}$denotes the projection onto $R_{+}^{n}$. It has attracted much attention because of its utility in reformulating complementarity and variational inequality problems as unconstrained minimization problems, see, e.g., Chen and Qi (2006), Facchinei and Kanzow (1997), Fukushima (1996), Kanzow and Fukushima (1998), Luo, Mangasarian, Ren and Solodov (1994), Peng (1997), Peng and Fukushima (1999), Solodov and Tseng (2000), Sun, Fukushima and Qi (1997), Tseng (1998), Tseng, Yamashita and Fukushima (1996), Yamashita and Fukushima (1995), Yamashita, Taji and Fukushima (1997). Mangasarian and Solodov (1993) showed that if the mapping $F$ is differentiable, so is the implicit Lagrangian. Yamashita and Fukushima (1995) showed that the implicit Lagrangian's gradient vanishes at each solution of NCP when the Jacobian is positive definite at this solution. Facchinei and Kanzow (1997) improved the result in (Yamashita and Fukushima 1995) and gave a necessary and sufficient condition for the stationary point of the implicit Lagrangian to be a solution of NCP. Peng (1997) extended the implicit Lagrangian to the variational inequality problem (VIP) and showed that the implicit Lagrangian can be represented as the difference of two regularized gap functions proposed by Fukushima (1992) and Auchmuty (1989) independently. Yamashita, Taji and Fukushima (1997) extended the results of Peng (1997) and studied various properties of the D-gap function

$$
g_{\alpha \beta}:=f_{\alpha}(x)-f_{\beta}(x)
$$


where $\alpha$ and $\beta$ are arbitrary positive parameters with $\alpha<\beta$, and $f_{\alpha}$ is the regularized gap function defined by

$$
f_{\alpha}(x):=\left\langle\alpha F(x), x-y_{\alpha}(x)\right\rangle-\frac{1}{2}\left\|x-y_{\alpha}(x)\right\|^{2}
$$

with $y_{\alpha}(x)=\prod_{X}(x-\alpha F(x))$ and $\prod_{X}(\cdot)$ being the projection operator onto the constraint set $X$ of VIP. Tseng (1998) and Tseng, Yamashita and Fukushima (1996) extended the implicit Lagrangian for NCP to the semi-definite and the generalized nonlinear complementarity problems, respectively. They also showed that the extended functions retain most of the nice properties of the implicit Lagrangian for NCP. In addition, several solution methods based on the D-gap function have been proposed; see, e.g., (Kanzow and Fukushima 1998, Peng and Fukushima 1999, Solodov and Tseng 2000, Sun, Fukushima and Qi 1997).

Motivated by these developments, we establish in this paper a vector-valued implicit Lagrangian for SCCP by utilizing the Jordan-algebraic structure. It not only provides a unified formula for the existing implicit Lagrange functions (Mangasarian and Solodov 1993, Tseng 1998), but also is of the vector-valued form which allows us to establish a reformulation of problem (1.1) as a smooth system of nonlinear equations. In particular, in Section 3 we show that it is a continuously differentiable complementarity function ( $C$-function) for SCCP and whose Jacobian is strongly semismooth, and hence it certainly can be combined with nonsmooth $C$ functions to obtain algorithms with fast convergent rates. Here, a function $\Phi: \mathcal{J} \times \mathcal{J} \rightarrow \mathcal{J}$ is said to be a $C$-function for $S C C P$ (see, e.g., Facchinei and Pang 2003, Isac 2000) if it satisfies

$$
\Phi(x, y)=0 \Longleftrightarrow x \in K, y \in K,\langle x, y\rangle=0 .
$$

In this case, $\Phi(x, y)=0$ is called the reformulation equation of the complementarity condition of problem (1.1). The concept of C-function for SCCP is in essence different from the one of the merit function for SCCP, where a function $\Psi: \mathcal{J} \times \mathcal{J} \rightarrow R$ is said to be a merit function for $\operatorname{SCCP}$ (see, e.g., Fukushima 1996) if $\Psi(x, y) \geq 0$ for any $(x, y) \in \mathcal{J} \times \mathcal{J}$, and $\Psi(x, y)=0$ if and only if $(x, y)$ is a solution to the complementarity condition of problem (1.1). In this case, the complementarity condition is equivalent to the unconstrained minimization problem

$$
\min _{(x, y) \in \mathcal{J} \times \mathcal{J}} \Psi(x, y)
$$

with zero optimal value. The concept of C-function for SCCP is also different from the one for NCP, because a $C$-function $\phi\left(R^{2} \rightarrow R\right)$ for NCP, which is also called the NCP function (see, e.g., Sun and Qi 1999), is a real-valued function which satisfies

$$
\phi(a, b)=0 \Longleftrightarrow a \geq 0, b \geq 0, a b=0
$$

By using the proposed vector-valued implicit Lagrangian, in Section 4 we introduce a realvalued implicit Lagrangian which can be regarded as a direct extension of those presented in (Mangasarian and Solodov 1993, Tseng 1998), and show that it possesses some interesting properties. In Section 5, we develop a merit function based on the real-valued implicit Lagrangian for problem (1.1), and give a necessary and sufficient condition for the stationary point of the merit function to be a solution of problem (1.1), which is weaker than requiring monotonicity. In Section 6, we introduce and give a characterization for the Cartesian $P$-property of the function $F$, and show the GUS-property (i.e., globally unique solvability) and a global error bound based on the implicit Lagrangian merit function for problem (1.1) with the uniform Cartesian P-property. Finally, we make some concluding remarks in Section 7. 
Notation: For a vector-valued function $E$, if it is differentiable, we let $E^{\prime}(x)$ denote the Jacobian operator of $E$ at a point $x$. Let $I$ be the identity operator from $\mathcal{J}$ into itself, i.e., $I x=x$ for all $x \in \mathcal{J}$. We say that a linear operator $A$ from $\mathcal{J}$ into itself is invertible (or nonsingular) if the equation $A x=0$ has a unique solution $x=0$. For a linear operator $A$ from $\mathcal{J}$ into itself, $A^{T}$ denotes the adjoint operator of $A$ in the sense of $\left\langle y, A^{T} z\right\rangle=\langle A y, z\rangle$ for all $y, z \in \mathcal{J}$.

\section{Preliminaries}

In this section, we first mention some basic concepts and results on Euclidean Jordan algebras. Koecher (1999) and Faraut and Korányi (1994) provided comprehensive treatments of Euclidean Jordan algebras. Excellent summaries can be found in the articles (Faybusovich 1997, Gowda, Sznajder and Tao 2004, Schmieta and Alizadeh 2003, Tao and Gowda 2005). We then introduce the concept of cone of a point, based on the convex hull and positive cone of a set, which will play an important role in the stationary point analysis in Section 5 of this paper.

A Euclidean Jordan algebra is a triple $(\mathcal{J},\langle\cdot, \cdot\rangle, \circ)$, where $(\mathcal{J},\langle\cdot, \cdot\rangle)$ is a finite-dimensional inner product space over real field $R$ and $(x, y) \rightarrow x \circ y: \mathcal{J} \times \mathcal{J} \rightarrow \mathcal{J}$ is a bilinear mapping which satisfies the following conditions:

(1) $x \circ y=y \circ x$ for all $x, y \in \mathcal{J}$,

(2) $x \circ\left(x^{2} \circ y\right)=x^{2} \circ(x \circ y)$ for all $x, y \in \mathcal{J}$ where $x^{2}:=x \circ x$ and

(3) $\langle x \circ y, z\rangle=\langle x, y \circ z\rangle$ for all $x, y, z \in \mathcal{J}$.

We call $x \circ y$ the Jordan product of $x$ and $y$. In addition, we assume that there is an element $e$ such that $x \circ e=e \circ x=x$ for all $x \in \mathcal{J}$, which is called the identity element in $\mathcal{J}$. Define the set of squares as $K:=\left\{x^{2}: x \in \mathcal{J}\right\}$. It is well known that $K$ is a symmetric cone. That is, $K$ is a self-dual closed convex cone with nonempty interior and for any two elements belonging to its interior $x, y \in \operatorname{int}(K)$, there exists an invertible linear transformation $\Gamma: \mathcal{J} \rightarrow \mathcal{J}$ such that $\Gamma(K)=K$ and $\Gamma(x)=y$.

An element $c \in \mathcal{J}$ is idempotent if $c^{2}=c \neq 0$. It is also primitive if it cannot be written as the sum of two idempotents. A complete system of orthogonal idempotents is a finite set $\left\{c_{1}, c_{2}, \cdots, c_{k}\right\}$ of idempotents with $c_{i} \circ c_{j}=0(i \neq j)$ and $\sum_{i=1}^{k} c_{i}=e$. A complete system of orthogonal primitive idempotents is called a Jordan frame of $\mathcal{J}$.

A classical example of Euclidean Jordan algebras is $R^{n}$ with the (usual) inner product and Jordan product defined respectively by

$$
\langle x, y\rangle:=\sum_{i=1}^{n} x_{i} y_{i} \text { and } \quad x \circ y:=x * y,
$$

where $x_{i}$ denotes the $i$ th component of $x$, and $x * y$ denotes the componentwise product of vectors $x$ and $y$. The identity element is the all ones vector, i.e., $e=(1, \cdots, 1)^{T}$. The set $\left\{e_{1}, \cdots, e_{n}\right\}$ is a unique Jordan frame in $R^{n}$ where $e_{i}$ is the $i$ th unit vector. A popular example of Euclidean Jordan algebras is $S^{n}$, where $S^{n}$ denotes the set of all $n \times n$ real symmetric matrices with the inner product and Jordan product defined respectively by

$$
\langle X, Y\rangle:=\operatorname{Trace}(X Y) \text { and } X \circ Y:=(X Y+Y X) / 2 .
$$

In this setting, the cone of squares $S_{+}^{n}$ is the set of all positive semidefinite matrices in $S^{n}$, and the identity element is the identity matrix $I$. The set $\left\{E_{1}, \cdots, E_{n}\right\}$ is a Jordan frame in 
$S^{n}$ where $E_{i}$ is the diagonal matrix with 1 in the $(i, i)$-slot and zeros everywhere else for every $i \in\{1,2, \cdots, n\}$. There are uncountably many Jordan frames for this example (any orthogonal system of $n$ elements of $S^{n}$ will do).

We below state the important spectral decomposition theorem for Euclidean Jordan algebras.

Theorem 2.1 (Spectral Decomposition Type II (Faraut and Korányi 1994, Theorem III.1.2)) Let $\mathcal{J}$ be a Euclidean Jordan algebra of rank $r$. Then for every vector $x \in \mathcal{J}$ there exists a Jordan frame $\left\{c_{1}, c_{2}, \cdots, c_{r}\right\}$ and real numbers $\lambda_{1}(x), \lambda_{2}(x), \cdots, \lambda_{r}(x)$, the eigenvalues of $x$, such that

$$
x=\lambda_{1}(x) c_{1}+\lambda_{2}(x) c_{2}+\cdots+\lambda_{r}(x) c_{r} .
$$

We call (2.1) the spectral decomposition of $x$.

Letting $q: R \rightarrow R$ be a real-valued function, we define a vector-valued function associated with the Euclidean Jordan algebra, which is called the Löwner function (operator) by Sun and Sun (2008):

$$
Q(x):=\sum_{j=1}^{r} q\left(\lambda_{j}(x)\right) c_{j}=q\left(\lambda_{1}(x)\right) c_{1}+q\left(\lambda_{2}(x)\right) c_{2}+\cdots+q\left(\lambda_{r}(x)\right) c_{r} .
$$

When $q(t)$ is taken as $t_{+}:=\max \{0, t\}, t_{-}:=\min \{0, t\}$, or $|t|:=t_{+}-t_{-}(t \in R)$, the Löwner function becomes the metric projection function

$$
x_{+}:=\sum_{i=1}^{r}\left(\lambda_{i}(x)\right)_{+} c_{i}, \quad x_{-}:=\sum_{i=1}^{r}\left(\lambda_{i}(x)\right)_{-} c_{i}, \text { or }|x|:=\sum_{i=1}^{r}\left|\lambda_{i}(x)\right| c_{i} .
$$

Note that $x \in K$ if and only if $\lambda_{i}(x) \geq 0(i=1,2, \cdots, r)$. It is easy to verify that

$$
x_{+} \in K, x_{-}=-(-x)_{+} \in(-K) .
$$

In other words, $x_{+}$is the projection of $x$ onto $K, x_{-}$is the projection of $x$ onto $(-K)$. Moreover, we can easily observe that

$$
x_{+} \circ x_{-}=0, x=x_{+}+x_{-}, \text {and }|x|=x_{+}-x_{-} .
$$

An important concept in the theory of Euclidean Jordan algebras is the Peirce decomposition which is stated as follows. Let $\left\{c_{1}, c_{2}, \cdots, c_{r}\right\}$ be a Jordan frame of $\mathcal{J}$. For $i, j \in\{1,2, \cdots, r\}$, define the subspaces

$$
J_{i i}:=\left\{y \in \mathcal{J}: y \circ c_{i}=y\right\}, \text { and } J_{i j}:=\left\{y \in \mathcal{J}: y \circ c_{i}=\frac{1}{2} y=y \circ c_{j}\right\}, i \neq j .
$$

Then from Theorem IV.2.1 in Faraut and Korányi (1994), we have the following result.

Theorem 2.2 Let $\mathcal{J}$ be a Euclidean Jordan algebra of rank $r$ and $\left\{c_{1}, c_{2}, \cdots, c_{r}\right\}$ be a given Jordan frame in $\mathcal{J}$. Then space $\mathcal{J}$ is the orthogonal direct sum of spaces $J_{i j}(i \leq j)$. Furthermore,

(1) $J_{i j} \circ J_{i j} \subseteq J_{i i}+J_{j j}$;

(2) $J_{i j} \circ J_{j k} \subseteq J_{i k}$, if $i \neq k$;

(3) $J_{i j} \circ J_{k l}=\{0\}$, if $\{i, j\} \cap\{k, l\}=\varnothing$. 
In the Euclidean Jordan algebra $\mathcal{J}$, for $x \in \mathcal{J}$, we define the corresponding Lyapunov transformation $L(x): \mathcal{J} \rightarrow \mathcal{J}$ by $L(x) y=x \circ y$ for all $y \in \mathcal{J}$. We say two elements $x, y \in \mathcal{J}$ operator commute if $L(x) L(y)=L(y) L(x)$. Lemma X.2.2 in Faraut and Korányi (1994) shows that the elements $x, y \in \mathcal{J}$ operator commute if and only if they share a common Jordan frame. Therefore, for a given Jordan frame $\left\{c_{1}, c_{2}, \cdots, c_{r}\right\}$, we have that $c_{i}, c_{j}$ operator commute, that is, $L\left(c_{i}\right) L\left(c_{j}\right)=L\left(c_{j}\right) L\left(c_{i}\right)$ for any $i, j \in\{1,2, \cdots, r\}$. Note that this is a generalization of a well-known fact for symmetric matrices.

The following proposition summarizes some equivalent reformulations related to the complementarity condition of problem (1.1).

Proposition 2.3 (Gowda, Sznajder and Tao 2004, Proposition 6) Let $K$ be the symmetric cone in $\mathcal{J}$. For $x, y \in \mathcal{J}$ and $\mu \in R$, the following conditions are equivalent:

(a) $x \in K, \quad y \in K$, and $\langle x, y\rangle=0$;

(b) $x \in K, \quad y \in K$, and $x \circ y=0$;

(c) $x+y \in K$, and $x \circ y=0$;

(d) $x-(x-\mu y)_{+}=0$ for any fixed $\mu>0$;

(e) $x+y-\sqrt{x^{2}+y^{2}}=0$, where $\sqrt{z}$ is the function given by (2.2) with $q(t)=\sqrt{t}$.

In each case, the elements $x$ and $y$ operator commute.

For any $x \in \mathcal{J}$, from Theorem 2.1 we have $x=\lambda_{1}(x) c_{1}(x)+\lambda_{2}(x) c_{2}(x)+\cdots+\lambda_{r}(x) c_{r}(x)$, where $\left\{c_{1}(x), c_{2}(x), \cdots, c_{r}(x)\right\}$ is a Jordan frame of $\mathcal{J}$. Define the subspaces

$$
J_{i i}(x):=\left\{z: z \circ c_{i}(x)=z\right\}, \quad J_{i j}(x):=\left\{z: z \circ c_{i}(x)=\frac{1}{2} z=z \circ c_{j}(x)\right\}, i \neq j .
$$

We obtain from Theorem 2.2 that $\lambda_{i}(x) c_{i}(x) \in J_{i i}(x)$ and for any $z \in J_{i j}(x)(i \neq j)$,

$$
\langle x, z\rangle=0 .
$$

Let us define a set $C:=\left\{\lambda_{1}(x) c_{1}(x), \lambda_{2}(x) c_{2}(x), \cdots, \lambda_{r}(x) c_{r}(x)\right\}$. Similar to the definition of convex hull $\operatorname{conv} C$ specified by

$$
\operatorname{conv} C:=\left\{\sum_{i=1}^{r} \theta_{i} \lambda_{i}(x) c_{i}(x): \sum_{i=1}^{r} \theta_{i}=1, \theta_{i} \geq 0, i=1,2, \cdots, r\right\},
$$

we can define the cone of a point $x$ with respect to $\left\{c_{1}(x), c_{2}(x), \cdots, c_{r}(x)\right\}$ as

$$
\operatorname{Cone}^{\left\{c_{1}(x), c_{2}(x), \cdots, c_{r}(x)\right\}}(x):=\left\{\sum_{i=1}^{r} \theta_{i} \lambda_{i}(x) c_{i}(x): \theta_{i} \geq 0, i=1,2, \cdots, r\right\} .
$$

Thus, we define the cone of a point $x$ as

$$
\text { Cone }(x):=\operatorname{conv}\left\{\operatorname{Cone}^{\left\{c_{1}(x), c_{2}(x), \cdots, c_{r}(x)\right\}}(x):\left\{c_{1}(x), c_{2}(x), \cdots, c_{r}(x)\right\} \in \mathcal{C}(x)\right\},
$$

where $\mathcal{C}(x)$ is the set consisting of all Jordan frames in the spectral decomposition of $x$.

This concept possesses the following features.

Proposition 2.4 The cone Cone $(x)$ is convex. Moreover, for any $w \in \operatorname{Cone}(x)$, we have

$$
\langle w, x\rangle \geq 0 \text {. }
$$


Proof. It is clear that the cone Cone $(x)$ is convex. By Carathéodory Theorem in Rockafellar and Wets (2004), for any $w \in \operatorname{Cone}(x)$, there exist elements $w_{i} \in \operatorname{Cone}^{\left\{c_{1}^{i}(x), c_{2}^{i}(x), \cdots, c_{r}^{i}(x)\right\}}(x)(i \in$ $\{1, \cdots, n\})$ such that

$$
w=\sum_{i=0}^{n} \mu_{i} w_{i}, \quad \text { with } \quad \mu_{i} \geq 0, \quad \sum_{i=0}^{n} \mu_{i}=1,
$$

where $\left\{c_{1}^{i}(x), c_{2}^{i}(x), \cdots, c_{r}^{i}(x)\right\} \in \mathcal{C}(x)$ and $n=\operatorname{dim}(\mathcal{J})$. It is enough to only verify that $\left\langle w_{i}, x\right\rangle \geq$ 0 for any $i \in\{1, \cdots, n\}$. In fact, by $(2.7)$ and taking $w_{i}=\sum_{j=1}^{r} \theta_{j} \lambda_{j}^{i}(x) c_{j}(x)$ with $\theta_{j} \geq 0$, we have

$$
\left\langle w_{i}, x\right\rangle=\sum_{j=1}^{r} \theta_{j}\left(\lambda_{j}^{i}(x)\right)^{2}\left\langle c_{j}^{i}(x), c_{j}^{i}(x)\right\rangle \geq 0,
$$

where the equality and inequality follow from the definition of the Jordan frame and the fact that $\left\{c_{1}^{i}(x), c_{2}^{i}(x), \cdots, c_{r}^{i}(x)\right\}$ forms a Jordan frame of $\mathcal{J}$.

When the set $\mathcal{C}(x)$ is singleton, i.e. the Jordan frame in the spectral decomposition of $x$ is unique, we have the following result.

Proposition 2.5 For $x \in \mathcal{J}$, suppose that $x=\lambda_{1}(x) c_{1}(x)+\lambda_{2}(x) c_{2}(x)+\cdots+\lambda_{r}(x) c_{r}(x)$ with $\mathcal{C}(x)=\left\{c_{1}(x), c_{2}(x), \cdots, c_{r}(x)\right\}$, and let $J_{i j}(x)$ be given by (2.5). Then Cone $(x)$ is a polyhedral cone in $\mathcal{J}$, and for any $w \in \operatorname{Cone}(x), z \in J_{i j}(x)$ with $i \neq j$, we have

$$
\langle w, x\rangle \geq 0, \quad\langle w, z\rangle=0 .
$$

Furthermore, $\langle w, v\rangle \geq 0$ for all $w, v \in \operatorname{Cone}(x)$.

Proof. It is obvious from Theorem 3.52 in Rockafellar and Wets $(2004)$ that Cone $(x)$ is a polyhedral cone. At the same time, it follows from (2.6) and the definition of Cone $(x)$ that $\langle w, z\rangle=0$ for any $w \in$ Cone $(x)$ and $z \in J_{i j}(x)(i \neq j)$. Finally, it follows from Proposition 2.4 that $\langle w, x\rangle \geq 0$ for any $w \in \operatorname{Cone}(x)$.

We end this section with two fundamental concepts related to the mapping $F: \mathcal{J} \rightarrow \mathcal{J}$. In the sequel, we say that $F$ is monotone if

$$
\langle x-y, F(x)-F(y)\rangle \geq 0, \quad \forall(x, y) \in \mathcal{J} \times \mathcal{J},
$$

and $F$ is strongly monotone with modulus $\mu>0$ if

$$
\langle x-y, F(x)-F(y)\rangle \geq \mu\|x-y\|^{2}, \quad \forall(x, y) \in \mathcal{J} \times \mathcal{J} .
$$

\section{Vector-Valued Implicit Lagrangian}

In this section, we shall introduce the vector-valued implicit Lagrangian for complementarity condition $x \in K, y \in K,\langle x, y\rangle=0$ of problem (1.1), and show mainly that it is a continuously differentiable C-function for SCCP and whose Jacobian is strongly semismooth.

For any fixed $\alpha>0$ and $\alpha \neq 1$, define the vector-valued implicit Lagrangian $\Phi_{\alpha}: \mathcal{J} \times \mathcal{J} \rightarrow \mathcal{J}$ as

$$
\Phi_{\alpha}(x, y):=x \circ y+\frac{1}{2 \alpha}\left\{\left[(x-\alpha y)_{+}\right]^{2}-x^{2}+\left[(y-\alpha x)_{+}\right]^{2}-y^{2}\right\} .
$$


Notice that in the above definition, we do not let $\alpha=1$. This is because when $\alpha=1$, one has for any $x, y \in \mathcal{J}$,

$$
\begin{aligned}
\Phi_{\alpha}(x, y) & =x \circ y+\frac{1}{2}\left\{\left[(x-y)_{+}\right]^{2}-x^{2}+\left[(y-x)_{+}\right]^{2}-y^{2}\right\} \\
& =x \circ y+\frac{1}{2}\left\{\left[(x-y)_{+}\right]^{2}-x^{2}+\left[(x-y)_{-}\right]^{2}-y^{2}\right\} \\
& =x \circ y+\frac{1}{2}\left\{(x-y)^{2}-x^{2}-y^{2}\right\} \\
& =0,
\end{aligned}
$$

where the second and third equalities come from (2.3) and (2.4).

It is clear that when $\mathcal{J}=R^{n}$ and $K=R_{+}^{n}$, the function $\Phi_{\alpha}$ becomes the implicit Lagrangian for NCP by Mangasarian and Solodov (1993) in the vector-valued form. So, the proposed function is an extension of the previous one. To the best of our knowledge, this extension is significant, since $\Phi_{\alpha}$ also provide the first vector-valued implicit Lagrangian for SOCCP as well as SDCP.

In order to built towards our main result, we first give some notations and lemmas. For any fixed $\alpha>0$ and $\alpha \neq 1$, let us define the function

$$
H_{\alpha}(x, y):=(x-\alpha y)_{+}, \quad(x, y) \in \mathcal{J} \times \mathcal{J},
$$

i.e., $H_{\alpha}(x, y)$ is the closest point in $K$ to the point $(x-\alpha y)$. Hence, it is the unique solution of the following problem:

$$
\min _{z \in K}\langle\alpha y, z-x\rangle+\frac{1}{2}\langle z-x, z-x\rangle .
$$

By using (3.2), we can define the vector-valued function

$$
G(x, y, \alpha):=-\alpha y \circ\left(H_{\alpha}(x, y)-x\right)-\frac{1}{2}\left(H_{\alpha}(x, y)-x\right)^{2} .
$$

It is easy to observe that the function $G$ generalizes $f_{\alpha}$ given by (1.6) in the sense that $f_{\alpha}(x)=$ $\langle e, G(x, y, \alpha)\rangle$ for $x, y \in R^{n}$. So, we call it the vector-valued regularized gap function for SCCP. The following proposition shows that the vector-valued implicit Lagrangian can be represented as the difference of two vector-valued regularized gap functions, and has nice symmetry with respect to $\alpha$.

Proposition 3.1 Let $1 \neq \alpha>0$ and $\Phi_{\alpha}(x, y), G(x, y, \alpha)$ be given by (3.1) and (3.4) respectively. Then,

$$
\Phi_{\alpha}(x, y)=\frac{1}{\alpha} G(x, y, \alpha)-\alpha G\left(x, y, \frac{1}{\alpha}\right) .
$$

Moreover, $\Phi_{\alpha}(x, y)=-\Phi_{\frac{1}{\alpha}}(x, y), \forall x, y \in \mathcal{J}$.

We also call $\Phi_{\alpha}$ the vector-valued D-gap function for SCCP.

Proof. By properties (2.3) and (2.4) of the projection,

$$
(y-\alpha x)_{+}+(y-\alpha x)_{-}=y-\alpha x .
$$

Hence, we have

$$
(\alpha x-y)_{+}=-(y-\alpha x)_{-}=(y-\alpha x)_{+}-y+\alpha x .
$$


Combining (3.1)-(3.4) and (3.6), for any fixed $\alpha>0$ and $\alpha \neq 1$, we obtain

$$
\begin{aligned}
& \frac{1}{\alpha} G(x, y, \alpha)-\alpha G\left(x, y, \frac{1}{\alpha}\right) \\
= & -y \circ\left[H_{\alpha}(x, y)-x\right]-\frac{1}{2 \alpha}\left[H_{\alpha}(x, y)-x\right]^{2} \\
& +y \circ\left[H_{\frac{1}{\alpha}}(x, y)-x\right]+\frac{\alpha}{2}\left[H_{\frac{1}{\alpha}}(x, y)-x\right]^{2} \\
= & -y \circ\left[(x-\alpha y)_{+}-x\right]-\frac{1}{2 \alpha}\left[(x-\alpha y)_{+}-x\right]^{2} \\
& +y \circ\left[\frac{1}{\alpha}(\alpha x-y)_{+}-x\right]+\frac{\alpha}{2}\left[\frac{1}{\alpha}(\alpha x-y)_{+}-x\right]^{2} \\
= & -y \circ\left[(x-\alpha y)_{+}-x\right]-\frac{1}{2 \alpha}\left[(x-\alpha y)_{+}-x\right]^{2} \\
& +y \circ\left\{\frac{1}{\alpha}\left[(y-\alpha x)_{+}-y+\alpha x\right]-x\right\}+\frac{\alpha}{2}\left\{\frac{1}{\alpha}\left[(y-\alpha x)_{+}-y+\alpha x\right]-x\right\}^{2} \\
= & -y \circ\left[(x-\alpha y)_{+}-x\right]-\frac{1}{2 \alpha}\left[(x-\alpha y)_{+}-x\right]^{2} \\
& +\frac{1}{\alpha} y \circ\left[(y-\alpha x)_{+}-y\right]+\frac{1}{2 \alpha}\left[(y-\alpha x)_{+}-y\right]^{2} \\
= & x \circ y-y \circ(x-\alpha y)_{+}-\frac{1}{2 \alpha}\left[(x-\alpha y)_{+}^{2}+x^{2}-2 x \circ(x-\alpha y)_{+}\right] \\
& +\frac{1}{\alpha} y \circ(y-\alpha x)_{+}-\frac{1}{\alpha} y^{2}+\frac{1}{2 \alpha}\left[(y-\alpha x)_{+}^{2}+y^{2}-2 y \circ(y-\alpha x)_{+}\right] \\
= & x \circ y+\frac{1}{2 \alpha}\left\{\left[(x-\alpha y)_{+}\right]^{2}-x^{2}+\left[(y-\alpha x)_{+}\right]^{2}-y^{2}\right\} \\
= & \Phi_{\alpha}(x, y),
\end{aligned}
$$

where the first equality follows from (3.4); the second equality follows from (3.2) and $\left(x-\frac{1}{\alpha} y\right)_{+}=$ $\frac{1}{\alpha}(\alpha x-y)_{+}$; the third equality holds by (3.6); the fourth and fifth equalities hold immediately by direct calculation; finally, the second to last equality follows from the fact $(x-\alpha y) \circ(x-\alpha y)_{+}=$ $\left[(x-\alpha y)_{+}\right]^{2}$ by $(2.4)$.

The second conclusion of the proposition is an obvious consequence of (3.5).

The following theorem tells us that the vector-valued implicit Lagrangian is a C-function for SCCP.

Theorem 3.2 Let $\mathcal{J}$ be a Euclidean Jordan algebra of rank $r$, and $K$ be the symmetric cone in $\mathcal{J}$. For $x, y \in \mathcal{J}$, the following statements are equivalent:

(a) $x \in K, \quad y \in K$, and $x \circ y=0$.

(b) $\Phi_{\alpha}(x, y)=x \circ y+\frac{1}{2 \alpha}\left\{\left[(x-\alpha y)_{+}\right]^{2}-x^{2}+\left[(y-\alpha x)_{+}\right]^{2}-y^{2}\right\}=0$ for any $1 \neq \alpha>0$.

Proof " $(a) \Rightarrow(b)$ ". Since (a) holds, the elements $x, y$ operator commute by Proposition 2.3. Thus, there is a Jordan frame $\left\{e_{1}, e_{2}, \cdots, e_{r}\right\}$ such that

$$
x=\sum_{i=1}^{r} x_{i} e_{i}, y=\sum_{i=1}^{r} y_{i} e_{i} .
$$


So, $x \circ y=\sum_{i=1}^{r} x_{i} y_{i} e_{i}$, and (a) implies that $x_{i} \geq 0, y_{i} \geq 0$ and $x_{i} y_{i}=0$ for all $i=1,2, \cdots, r$. Then for any fixed $\alpha>0$ and all $i=1,2, \cdots, r$, we have $\left(x_{i}-\alpha y_{i}\right)_{+}=x_{i},\left(y_{i}-\alpha x_{i}\right)_{+}=y_{i}$. This implies from (3.7) that

$$
(x-\alpha y)_{+}=\sum_{i=1}^{r}\left(x_{i}-\alpha y_{i}\right)_{+} e_{i}=\sum_{i=1}^{r} x_{i} e_{i}=x,
$$

and

$$
(y-\alpha x)_{+}=\sum_{i=1}^{r}\left(y_{i}-\alpha x_{i}\right)_{+} e_{i}=\sum_{i=1}^{r} y_{i} e_{i}=y .
$$

Hence for any fixed $\alpha>0$,

$$
\Phi_{\alpha}(x, y)=x \circ y+\frac{1}{2 \alpha}\left\{x^{2}-x^{2}+y^{2}-y^{2}\right\}=0,
$$

and (b) holds.

"(b) $\Rightarrow(a) "$. Suppose that $\Phi_{\alpha}(x, y)=0$ for $x, y \in \mathcal{J}$. Let us define $g(x, y, \alpha)$ to be the negative of the optimal value of problem (3.3), i.e.,

$$
g(x, y, \alpha):=-\left\langle\alpha y, H_{\alpha}(x, y)-x\right\rangle-\frac{1}{2}\left\langle H_{\alpha}(x, y)-x, H_{\alpha}(x, y)-x\right\rangle .
$$

By using (3.10), (3.4) and the properties of the Jordan product (see Section 2), we conclude

$$
\begin{aligned}
g(x, y, \alpha) & =-\left\langle e, \alpha y \circ\left(H_{\alpha}(x, y)-x\right)\right\rangle-\frac{1}{2}\left\langle e,\left(H_{\alpha}(x, y)-x\right)^{2}\right\rangle \\
& =-\left\langle e, \alpha y \circ\left(H_{\alpha}(x, y)-x\right)+\frac{1}{2}\left(H_{\alpha}(x, y)-x\right)^{2}\right\rangle \\
& =\langle e, G(x, y, \alpha)\rangle .
\end{aligned}
$$

To proceed with the verification, we below discuss two cases.

Case 1: $\alpha>1$. In this case, since $H_{\frac{1}{\alpha}}(x, y) \in K$ is a feasible solution of the closest point problem (3.3), we have

$$
g(x, y, \alpha) \geq-\left\langle\alpha y, H_{\frac{1}{\alpha}}(x, y)-x\right\rangle-\frac{1}{2}\left\langle H_{\frac{1}{\alpha}}(x, y)-x, H_{\frac{1}{\alpha}}(x, y)-x\right\rangle .
$$

By using (3.5), (3.11) and (3.12), direct calculation yields that for any fixed $\alpha>1$,

$$
\begin{aligned}
0 & =\left\langle e, \Phi_{\alpha}(x, y)\right\rangle \\
= & \left\langle e, \frac{1}{\alpha} G(x, y, \alpha)-\alpha G\left(x, y, \frac{1}{\alpha}\right)\right\rangle \\
= & \frac{1}{\alpha} g(x, y, \alpha)-\alpha g\left(x, y, \frac{1}{\alpha}\right) \\
\geq & \frac{1}{\alpha}\left[-\left\langle\alpha y, H_{\frac{1}{\alpha}}(x, y)-x\right\rangle-\frac{1}{2}\left\langle H_{\frac{1}{\alpha}}(x, y)-x, H_{\frac{1}{\alpha}}(x, y)-x\right\rangle\right] \\
& +\alpha\left[\left\langle\frac{1}{\alpha} y, H_{\frac{1}{\alpha}}(x, y)-x\right\rangle+\frac{1}{2}\left\langle H_{\frac{1}{\alpha}}(x, y)-x, H_{\frac{1}{\alpha}}(x, y)-x\right\rangle\right] \\
= & \frac{\alpha^{2}-1}{2 \alpha}\left\langle H_{\frac{1}{\alpha}}(x, y)-x, H_{\frac{1}{\alpha}}(x, y)-x\right\rangle \\
\geq & 0 .
\end{aligned}
$$

This implies that for any fixed $\alpha>1$,

$$
\left\langle H_{\frac{1}{\alpha}}(x, y)-x, H_{\frac{1}{\alpha}}(x, y)-x\right\rangle=0 .
$$


The desired conclusion follows immediately from (3.2) and Proposition 2.3 (d).

Case 2: $0<\alpha<1$. Similar to the proof of (3.12), we have for $H_{\alpha}(x, y) \in K$,

$$
g\left(x, y, \frac{1}{\alpha}\right) \geq-\left\langle\frac{1}{\alpha} y, H_{\alpha}(x, y)-x\right\rangle-\frac{1}{2}\left\langle H_{\alpha}(x, y)-x, H_{\alpha}(x, y)-x\right\rangle,
$$

from which we directly derive that for any fixed $0<\alpha<1$,

$$
\begin{aligned}
0 & =\left\langle e, \Phi_{\alpha}(x, y)\right\rangle \\
& =\frac{1}{\alpha} g(x, y, \alpha)-\alpha g\left(x, y, \frac{1}{\alpha}\right) \\
& \leq \frac{1}{\alpha}\left[-\left\langle\alpha y, H_{\alpha}(x, y)-x\right\rangle-\frac{1}{2}\left\langle H_{\alpha}(x, y)-x, H_{\alpha}(x, y)-x\right\rangle\right] \\
& +\alpha\left[\left\langle\frac{1}{\alpha} y, H_{\alpha}(x, y)-x\right\rangle+\frac{1}{2}\left\langle H_{\alpha}(x, y)-x, H_{\alpha}(x, y)-x\right\rangle\right] \\
& =\frac{\alpha^{2}-1}{2 \alpha}\left\langle H_{\alpha}(x, y)-x, H_{\alpha}(x, y)-x\right\rangle \\
& \leq 0 .
\end{aligned}
$$

This implies that for any fixed $\alpha \in(0,1)$,

$$
\left\langle H_{\alpha}(x, y)-x, H_{\alpha}(x, y)-x\right\rangle=0 .
$$

The desired conclusion follows.

Combining the above two cases, we complete the proof.

The function $g$ given by (3.10) is actually a special case of the (real-valued) regularized gap function in the setting of variational inequalities Fukushima (1992), and can be regarded as a consequence of the vector-valued regularized gap function $G$ given by (3.4).

It is well known that the implicit Lagrangian with $\alpha>1$ is nonnegative in the context of NCP. So, it is natural to ask whether the vector-valued implicit Lagrangian $\Phi_{\alpha}$ is in $K$ or not. Unfortunately, it fails to preserve this property, which is illustrated by the following example in the setting of the 3 -dimensional second-order cone (this also proves that such property does not hold for $S_{+}^{n}$ with $n \geq 2$ ).

Example. For any $x=\left(x_{1}, x_{2}\right)$ and $y=\left(y_{1}, y_{2}\right)$ in $R^{n}(n>1)$ with $x_{1}, y_{1} \in R$, we define the Jordan product of $x$ and $y$ as

$$
x \circ y=\left(\begin{array}{l}
x_{1} \\
x_{2}
\end{array}\right) \circ\left(\begin{array}{l}
y_{1} \\
y_{2}
\end{array}\right):=\left(\begin{array}{c}
\langle x, y\rangle \\
x_{1} y_{2}+y_{1} x_{2}
\end{array}\right) .
$$

As we know, $\left(R^{n},\langle\cdot, \cdot\rangle, \circ\right)$ forms a Euclidean Jordan algebra denoted by $\Lambda^{n}$. In this algebra, the second-order cone (SOC) $\Lambda_{+}^{n}$ is the cone of squares, i.e., $\Lambda_{+}^{n}=\left\{x^{2}: x \in \Lambda^{n}\right\}$ (see, e.g., Faraut and Korányi 1994).

Let $x=(1,1,0)^{T} \in \Lambda_{+}^{3}$ and $y=(1,0,1)^{T} \in \Lambda_{+}^{3}$. Then direct calculation yields

$$
x^{2}=\left(\begin{array}{l}
2 \\
2 \\
0
\end{array}\right), y^{2}=\left(\begin{array}{l}
2 \\
0 \\
2
\end{array}\right), x \circ y=\left(\begin{array}{l}
1 \\
1 \\
1
\end{array}\right), x-\alpha y=\left(\begin{array}{c}
1-\alpha \\
1 \\
-\alpha
\end{array}\right), y-\alpha x=\left(\begin{array}{c}
1-\alpha \\
-\alpha \\
1
\end{array}\right) .
$$

By the spectral factorization and the definition of the projection $(\cdot)_{+}$, we have

$$
(x-\alpha y)_{+}=\frac{1}{2}\left(1-\alpha+\sqrt{1+\alpha^{2}}\right)\left(\begin{array}{c}
\frac{1}{\sqrt{1+\alpha^{2}}} \\
\frac{-\alpha}{\sqrt{1+\alpha^{2}}}
\end{array}\right),(y-\alpha x)_{+}=\frac{1}{2}\left(1-\alpha+\sqrt{1+\alpha^{2}}\right)\left(\begin{array}{c}
1 \\
\frac{-\alpha}{\sqrt{1+\alpha^{2}}} \\
\frac{1}{\sqrt{1+\alpha^{2}}}
\end{array}\right)
$$


for any fixed $\alpha>0$ and $\alpha \neq 1$. Therefore,

$$
(x-\alpha y)_{+}^{2}+(y-\alpha x)_{+}^{2}=\left(\alpha^{2}+1-\alpha+\sqrt{1+\alpha^{2}}(1-\alpha)\right)\left(\begin{array}{c}
2 \\
\frac{1-\alpha}{\sqrt{1+\alpha^{2}}} \\
\frac{1-\alpha}{\sqrt{1+\alpha^{2}}}
\end{array}\right) .
$$

Taking $\alpha=2$, we obtain that

$$
\begin{aligned}
2 \alpha \Phi_{\alpha} & =2 \alpha x \circ y+\left[(x-\alpha y)_{+}\right]^{2}+\left[(y-\alpha x)_{+}\right]^{2}-\left(x^{2}+y^{2}\right) \\
& =\left(\begin{array}{l}
4 \\
4 \\
4
\end{array}\right)+(3-\sqrt{5})\left(\begin{array}{c}
2 \\
-\frac{1}{\sqrt{5}} \\
-\frac{1}{\sqrt{5}}
\end{array}\right)-\left(\begin{array}{l}
4 \\
2 \\
2
\end{array}\right) \\
& =\left(\begin{array}{c}
\frac{6 \sqrt{5}-10}{\sqrt{5}} \\
\frac{3 \sqrt{5}-3}{\sqrt{5}} \\
\frac{3 \sqrt{5}-3}{\sqrt{5}}
\end{array}\right) .
\end{aligned}
$$

Obviously, $\Phi_{\alpha} \notin \Lambda_{+}^{3}$ with $\alpha=2$ because $(6 \sqrt{5}-10)^{2}<2(3 \sqrt{5}-3)^{2}$.

In the rest of this section, we address the continuous differentiability of the vector-valued implicit Lagrangian. The following lemma can be easily proved using the results on differentiability and semismoothness of Löwner's operator by Sun and Sun (2008); hence its proof is omitted.

Lemma 3.3 Let $x, y \in \mathcal{J}$. Then the functions $x, x^{2}, x^{\mu}(\mu \geq 3), x_{+}^{2}$ and $x \circ y$ are continuously differentiable. Moreover,

(1) $(x)^{\prime}=L(e)$;

(2) $(x \circ x)^{\prime}=2 L(x)$;

(3) $(x \circ y)^{\prime}=(L(y) L(x))$;

(4) $\left(x_{+} \circ x_{+}\right)^{\prime} e=2 L\left(x_{+}\right) e$;

(5) $\left(x^{k}\right)^{\prime} e=k L\left(x^{k-1}\right)$ e for $k \in R$, where $x^{0}:=e$.

Remark. It is appropriate to point out that in general, (i) $\left(x_{+} \circ x_{+}\right)^{\prime} \neq 2 L\left(x_{+}\right)$; (ii) $\left(x^{\mu}\right)^{\prime} \neq \mu L\left(x^{\mu-1}\right)$ for $\mu \geq 3$. They are verified as follows.

Part (i): Consider the second-order cone $\Lambda_{+}^{3}$. Let $\bar{x}=(0,0, \sqrt{2})^{T} \in \Lambda^{3}$. By the spectral factorization and the definition of the projection $(\cdot)_{+}$, we have

$$
\bar{x}=\frac{\sqrt{2}}{2}\left(\begin{array}{l}
1 \\
0 \\
1
\end{array}\right)+\left(-\frac{\sqrt{2}}{2}\right)\left(\begin{array}{c}
1 \\
0 \\
-1
\end{array}\right), \quad \bar{x}_{+}=\frac{\sqrt{2}}{2}\left(\begin{array}{l}
1 \\
0 \\
1
\end{array}\right) .
$$

That is, the eigenvalues of $\bar{x}$ are $\lambda_{1}=\sqrt{2}$ and $\lambda_{2}=-\sqrt{2}$, and the corresponding Jordan frame is $\left\{c_{1}, c_{2}\right\}$ with $c_{1}=\frac{1}{2}(1,0,1)^{T}, c_{2}=\frac{1}{2}(1,0,-1)^{T}$. Note that for $x=\left(x_{1}, x_{2}, x_{3}\right)^{T} \in \Lambda^{3}$, the corresponding Lyapunov transformation is

$$
L(x)=\left(\begin{array}{ccc}
x_{1} & x_{2} & x_{3} \\
x_{2} & x_{1} & 0 \\
x_{3} & 0 & x_{1}
\end{array}\right)
$$


Thus, we have

$$
L\left(\bar{x}_{+}\right)=\left(\begin{array}{ccc}
\frac{\sqrt{2}}{2} & 0 & \frac{\sqrt{2}}{2} \\
0 & \frac{\sqrt{2}}{2} & 0 \\
\frac{\sqrt{2}}{2} & 0 & \frac{\sqrt{2}}{2}
\end{array}\right)
$$

Taking $\bar{h}=(1,1,0)^{T}$, we obtain

$$
2 L\left(\bar{x}_{+}\right) \bar{h}=2\left(\begin{array}{ccc}
\frac{\sqrt{2}}{2} & 0 & \frac{\sqrt{2}}{2} \\
0 & \frac{\sqrt{2}}{2} & 0 \\
\frac{\sqrt{2}}{2} & 0 & \frac{\sqrt{2}}{2}
\end{array}\right)\left(\begin{array}{l}
1 \\
1 \\
0
\end{array}\right)=\left(\begin{array}{c}
\sqrt{2} \\
\sqrt{2} \\
\sqrt{2}
\end{array}\right) .
$$

At the same time, for $\bar{h}=(1,1,0)^{T}$, using Theorem 13 in Sun and Sun (2008) with $q(t)=t_{+}^{2}$ we compute

$$
\begin{aligned}
\left(x_{+} \circ x_{+}\right)^{\prime} \bar{h} & =\sum_{j=1}^{2}\left(q^{[1]}(\lambda(x))\right)_{j j} \frac{\left\langle c_{j}, \bar{h}\right\rangle}{\left\|c_{j}\right\|^{2}} c_{j}+\sum_{1 \leq j<l \leq 2} 4\left(q^{[1]}(\lambda(x))\right)_{j l} c_{j} \circ\left(c_{l} \circ \bar{h}\right) \\
& =2\left(\lambda_{1}\right)_{+} \frac{\left\langle c_{1}, \bar{h}\right\rangle}{\left\|c_{1}\right\|^{2}} c_{1}+4\left(q^{[1]}(\lambda(x))\right)_{1,2} c_{1} \circ\left(c_{2} \circ \bar{h}\right) \\
& \left.=2(\sqrt{2}) \frac{1}{2}\left(\begin{array}{c}
1 \\
0 \\
1
\end{array}\right)+4 \frac{(\sqrt{2})^{2}-0}{\sqrt{2}-(-\sqrt{2})} \frac{1}{2}\left(\begin{array}{c}
1 \\
0 \\
1
\end{array}\right) \circ\left[\begin{array}{c}
1 \\
\frac{1}{2} \\
0 \\
-1
\end{array}\right) \circ\left(\begin{array}{c}
1 \\
1 \\
0
\end{array}\right)\right] \\
& =\sqrt{2}\left(\begin{array}{c}
1 \\
0 \\
1
\end{array}\right)+\frac{1}{\sqrt{2}}\left(\begin{array}{c}
1 \\
0 \\
1
\end{array}\right) \circ\left(\begin{array}{c}
1 \\
1 \\
-1
\end{array}\right)=\left(\begin{array}{c}
\sqrt{2} \\
\frac{1}{\sqrt{2}} \\
\sqrt{2}
\end{array}\right) .
\end{aligned}
$$

This together with (3.17) shows that $\left(x_{+} \circ x_{+}\right)^{\prime} \bar{h} \neq 2 L\left(x_{+}\right) \bar{h}$.

Part (ii): We first claim that generally

$$
L(x) L(x) \neq L\left(x^{2}\right), \text { for } x \in \mathcal{J} .
$$

In the same setting as Part (i), we have $\bar{h}^{2}=(2,2,0)^{T}$ and then

$$
L(\bar{h})=\left(\begin{array}{lll}
1 & 1 & 0 \\
1 & 1 & 0 \\
0 & 0 & 1
\end{array}\right), \quad L\left(\bar{h}^{2}\right)=\left(\begin{array}{lll}
2 & 2 & 0 \\
2 & 2 & 0 \\
0 & 0 & 2
\end{array}\right) .
$$

Therefore, direct calculation yields

$$
L(\bar{h}) L(\bar{h})=\left(\begin{array}{lll}
1 & 1 & 0 \\
1 & 1 & 0 \\
0 & 0 & 1
\end{array}\right)\left(\begin{array}{lll}
1 & 1 & 0 \\
1 & 1 & 0 \\
0 & 0 & 1
\end{array}\right)=\left(\begin{array}{lll}
2 & 2 & 0 \\
2 & 2 & 0 \\
0 & 0 & 1
\end{array}\right) \neq L\left(\bar{h}^{2}\right) .
$$

When $\mu=3$, we compute

$$
\begin{aligned}
(x+\Delta x)^{3}-x^{3} & =\left(x^{2}+2 x \circ \Delta x+(\Delta x)^{2}\right) \circ(x+\Delta x)-x^{3} \\
& =x^{2} \circ \Delta x+2(x \circ \Delta x) \circ x+2(x \circ \Delta x) \circ \Delta x+(\Delta x)^{2} \circ(x+\Delta x) \\
& =L\left(x^{2}\right) \Delta x+2 L(x) L(x) \Delta x+o(\|\Delta x\|) .
\end{aligned}
$$

This along with (3.18) means that in general, $\left(x^{3}\right)^{\prime}=L\left(x^{2}\right)+2 L(x) L(x) \neq 3 L\left(x^{2}\right)$. 
Theorem 3.4 Let $\Phi_{\alpha}(x, y)$ be defined by (3.1). Then $\Phi_{\alpha}(x, y)$ is continuously differentiable at any $(x, y) \in \mathcal{J} \times \mathcal{J}$ and has the following strongly semismooth Jacobian:

$$
\Phi_{\alpha}^{\prime}(x, y)=\left(\nabla_{x}^{T} \Phi_{\alpha}(x, y), \nabla_{y}^{T} \Phi_{\alpha}(x, y)\right)
$$

where

$$
\begin{aligned}
\nabla_{x}^{T} \Phi_{\alpha}(x, y) e & =\left\{L(y)+\frac{1}{\alpha}\left[L\left((x-\alpha y)_{+}\right)-L(x)-\alpha L\left((y-\alpha x)_{+}\right)\right]\right\} e \\
& =y+\frac{1}{\alpha}\left[(x-\alpha y)_{+}-x-\alpha(y-\alpha x)_{+}\right]
\end{aligned}
$$

and

$$
\begin{aligned}
\nabla_{y}^{T} \Phi_{\alpha}(x, y) e & =\left\{L(x)+\frac{1}{\alpha}\left[L\left((y-\alpha x)_{+}\right)-L(y)-\alpha L\left((x-\alpha y)_{+}\right)\right]\right\} e \\
& =x+\frac{1}{\alpha}\left[(y-\alpha x)_{+}-y-\alpha(x-\alpha y)_{+}\right] .
\end{aligned}
$$

Proof. The desired result follows immediately from Lemma 3.3 and the strong semismoothness of $(x)_{+}$(see Sun and Sun 2008).

Replacing the complementarity condition by $\Phi_{\alpha}(x, y)=0$, we can obtain a smooth equation reformulation of problem (1.1):

$$
\Theta(x, y):=\left[\begin{array}{c}
\Phi_{\alpha}(x, y) \\
y-F(x)
\end{array}\right]=0 .
$$

It has the following Jacobian operator:

$$
\Theta^{\prime}(x, y)=\left(\begin{array}{cc}
\nabla_{x}^{T} \Phi_{\alpha}(x, y) & \nabla_{y}^{T} \Phi_{\alpha}(x, y) \\
-F^{\prime}(x) & I
\end{array}\right) .
$$

Note that the Jacobian $\Theta^{\prime}(x, y)$ may be singular at each solution of problem (1.1). Therefore, a direct application of Newton's method for the equation (3.20) might have serious difficulties near a solution. However, we may use other numerical methods for (3.20), such as the Gauss-Newton method, the hybrid Newton-type method etc.. These methods all need a proper function to measure how far $\Phi_{\alpha}(x, y)$ is away from the origin. So, it is necessary to develop a real-valued implicit Lagrangian induced by the vector-valued implicit Lagrangian for SCCP.

\section{Real-Valued Implicit Lagrangian}

As an application of the proposed vector-valued implicit Lagrangian, we construct in this section a real-valued implicit Lagrangian for the complementarity condition of problem (1.1), and show that it is a continuously differentiable merit function for SCCP. Although some results (Theorems 4.1 and 4.3 below) are the special cases of Proposition 2.2 in Tseng, Yamashita and Fukushima (1996), here we take advantage of the Jordan algebra structure. For completeness, we have included the proofs.

For any fixed $\alpha>0$ and $\alpha \neq 1$, by using the $D$-gap function $\Phi_{\alpha}$ given by (3.1), we define the real-valued implicit Lagrangian $\varphi_{\alpha}: \mathcal{J} \times \mathcal{J} \rightarrow R$ by

$$
\varphi_{\alpha}(x, y):=\left\langle e, \Phi_{\alpha}(x, y)\right\rangle
$$


which, by simple Jordan product operation, can be rewritten as

$$
\varphi_{\alpha}(x, y)=\langle x, y\rangle+\frac{1}{2 \alpha}\left\{\left\|(x-\alpha y)_{+}\right\|^{2}-\|x\|^{2}+\left\|(y-\alpha x)_{+}\right\|^{2}-\|y\|^{2}\right\} .
$$

From (3.5) and (3.11), we also conclude that

$$
\varphi_{\alpha}(x, y)=\left\langle e, \frac{1}{\alpha} G(x, y, \alpha)-\alpha G\left(x, y, \frac{1}{\alpha}\right)\right\rangle=\frac{1}{\alpha} g(x, y, \alpha)-\alpha g\left(x, y, \frac{1}{\alpha}\right) .
$$

Observing the proof of Theorem 3.2, we know from (3.13) and (3.14) that the following theorem has been proved.

Theorem 4.1 Let $\mathcal{J}$ be a Euclidean Jordan algebra of rank $r, K$ be the symmetric cone in $\mathcal{J}$. Then for $x, y \in \mathcal{J}, \varphi_{\alpha}(x, y) \geq 0$ if $\alpha>1 ; \varphi_{\alpha}(x, y) \leq 0$ if $0<\alpha<1$. Moreover, the following statements are equivalent:

(a) $x \in K, \quad y \in K$, and $x \circ y=0$.

(b) $\varphi_{\alpha}(x, y)=\left\langle e, \Phi_{\alpha}(x, y)\right\rangle=0, \alpha>0, \alpha \neq 1$.

Thus, $\varphi_{\alpha}$ is a merit function for SCCP, which is also called the real-valued D-gap function for SCCP. It is easily seen from (4.1) and (4.2) that when $\mathcal{J}=R^{n}$ and $K=R_{+}^{n}$, the proposed function becomes the well-known implicit Lagrangian introduced by Mangasarian and Solodov (1993) as a merit function for NCP. When $\mathcal{J}=S^{n}$ and $K=S_{+}^{n}$, the proposed function becomes the well-known implicit Lagrangian proposed by Tseng (1998) as a merit function for SDCP. So, our function gives a unified generalization for such a class of merit functions.

The following lemma shows that the differentiability of the real-valued implicit Lagrangian depends on that of the vector-valued implicit Lagrangian.

Lemma 4.2 Let $\varphi_{\alpha}: \mathcal{J} \times \mathcal{J} \rightarrow R$ be defined by (4.1). Then, it is a continuously differentiable function, and its gradient is given by

$$
\nabla \varphi_{\alpha}(x, y)=\Phi_{\alpha}^{\prime}(x, y)^{T} e
$$

Proof. Noting that $\varphi_{\alpha}(x, y)$ is a composition of two functions $\langle e, z\rangle$ and $z:=\Phi_{\alpha}(x, y)$, the desired result follows immediately from Theorem 3.4.

Combining this lemma with (3.19), we have

$$
\nabla \varphi_{\alpha}(x, y):=\left(\begin{array}{c}
\nabla_{x} \varphi_{\alpha}(x, y) \\
\nabla_{y} \varphi_{\alpha}(x, y)
\end{array}\right)=\left(\begin{array}{c}
y+\frac{1}{\alpha}\left[(x-\alpha y)_{+}-x-\alpha(y-\alpha x)_{+}\right] \\
x+\frac{1}{\alpha}\left[(y-\alpha x)_{+}-y-\alpha(x-\alpha y)_{+}\right]
\end{array}\right) .
$$

Employing the gradient of $\varphi_{\alpha}$, we now present another equivalent reformulation of the complementarity condition.

Theorem 4.3 Let $\varphi_{\alpha}: \mathcal{J} \times \mathcal{J} \rightarrow R$ be given by (4.1). Then, the following statements are equivalent:

(a) $x \in K, \quad y \in K$, and $x \circ y=0$.

(b) $\nabla \varphi_{\alpha}(x, y)=0$. 
Proof. " $(a) \Rightarrow(b)$ " As in the proof of Theorem 3.2, we have (3.8) and (3.9), and hence from $(4.3)^{\prime}$,

$$
\nabla \varphi_{\alpha}(x, y)=\left(\begin{array}{c}
y+\frac{1}{\alpha}[x-x-\alpha y] \\
x+\frac{1}{\alpha}[y-y-\alpha x]
\end{array}\right)=0
$$

"(b) $\Rightarrow(a)$ " Since $\nabla \varphi_{\alpha}(x, y)=0$, by using $(4.3)^{\prime}$ we have

$$
\begin{aligned}
& y+\frac{1}{\alpha}\left[(x-\alpha y)_{+}-x-\alpha(y-\alpha x)_{+}\right]=0, \\
& x+\frac{1}{\alpha}\left[(y-\alpha x)_{+}-y-\alpha(x-\alpha y)_{+}\right]=0 .
\end{aligned}
$$

That is,

$$
\begin{gathered}
(x-\alpha y)_{+}-x+\alpha y=\alpha(y-\alpha x)_{+}, \\
-\alpha(x-\alpha y)_{+}+\alpha x-y=-(y-\alpha x)_{+} .
\end{gathered}
$$

Combining (4.4) with (4.5), we obtain that $\left(1-\alpha^{2}\right)\left[(x-\alpha y)_{+}-x\right]=0$. Noting that $\alpha>0$ and $\alpha \neq 1$, we have $x-(x-\alpha y)_{+}=0$. The desired conclusion follows from Proposition $2.3(\mathrm{~d})$.

Furthermore, we can obtain the following interesting properties of the real-valued implicit Lagrangian. They will be used in the unconstrained stationary point analysis in the next section.

Proposition 4.4 For all $x, y \in \mathcal{J}$, we have the following:

(1) $\left\langle\nabla_{x} \varphi_{\alpha}(x, y), \nabla_{y} \varphi_{\alpha}(x, y)\right\rangle \geq 0$

(2) $\left\langle\nabla_{x} \varphi_{\alpha}(x, y), \nabla_{y} \varphi_{\alpha}(x, y)\right\rangle=0 \Leftrightarrow \nabla \varphi_{\alpha}(x, y) \circ \nabla_{y} \varphi_{\alpha}(x, y)=0$;

(3) $\nabla_{x} \varphi_{\alpha}(0, y)=0$;

(4) $\nabla_{y} \varphi_{\alpha}(0, y) \in(-K)$ if $\alpha>1 ; \nabla_{y} \varphi_{\alpha}(0, y) \in K$ if $0<\alpha<1$.

Proof. Part (1): By $(4.3)^{\prime}$, direct calculation yields

$$
\begin{aligned}
& \left\langle\nabla_{x} \varphi_{\alpha}(x, y), \nabla_{y} \varphi_{\alpha}(x, y)\right\rangle \\
= & \frac{1}{\alpha^{2}}\left\langle(x-\alpha y)_{+}-x+\alpha y-\alpha(y-\alpha x)_{+},-\alpha(x-\alpha y)_{+}+\alpha x-y+(y-\alpha x)_{+}\right\rangle \\
= & \frac{1}{\alpha^{2}}\left\langle-(x-\alpha y)_{-}-\alpha(y-\alpha x)_{+},-\alpha(x-\alpha y)_{+}-(y-\alpha x)_{-}\right\rangle \\
= & \frac{1}{\alpha^{2}}\left[\left\langle-(x-\alpha y)_{-},-(y-\alpha x)_{-}\right\rangle\right]+\left\langle(y-\alpha x)_{+},(x-\alpha y)_{+}\right\rangle \\
\geq & 0,
\end{aligned}
$$

where the first equality follows from $(4.3)^{\prime}$; the second equality follows from $x-\alpha y=(x-$ $\alpha y)_{+}+(x-\alpha y)_{-}$and $y-\alpha x=(y-\alpha x)_{+}+(y-\alpha x)_{-}$by (2.4); the third equality follows from $\left\langle(x-\alpha y)_{+},(x-\alpha y)_{-}\right\rangle=0$ and $\left\langle(y-\alpha x)_{+},(y-\alpha x)_{-}\right\rangle=0$; finally, the inequality follows from the definition of the symmetric cone.

Part (2): Suppose that $\nabla \varphi_{\alpha}(x, y) \circ \nabla_{y} \varphi_{\alpha}(x, y)=0$. Then taking the inner product of both sides with the identity element $e$ gives

$$
0=\left\langle e, \nabla_{x} \varphi_{\alpha}(x, y) \circ \nabla_{y} \varphi_{\alpha}(x, y)\right\rangle=\left\langle\nabla_{x} \varphi_{\alpha}(x, y), \nabla_{y} \varphi_{\alpha}(x, y)\right\rangle .
$$

Conversely, suppose that $\left\langle\nabla_{x} \varphi_{\alpha}(x, y), \nabla_{y} \varphi_{\alpha}(x, y)\right\rangle=0$. Then from the proof of Part (1), we obtain

$$
\left\langle-(x-\alpha y)_{-},-(y-\alpha x)_{-}\right\rangle=0, \quad\left\langle(y-\alpha x)_{+},(x-\alpha y)_{+}\right\rangle=0 .
$$


Since $-(x-\alpha y)_{-} \in K,-(y-\alpha x)_{-} \in K,(y-\alpha x)_{+} \in K,(x-\alpha y)_{+} \in K$, from Proposition 2.3 we derive immediately that

$$
\left[-(x-\alpha y)_{-}\right] \circ\left[-(y-\alpha x)_{-}\right]=0, \quad(y-\alpha x)_{+} \circ(x-\alpha y)_{+}=0 .
$$

Thus, the direct calculation yields $\nabla \varphi_{\alpha}(x, y) \circ \nabla_{y} \varphi_{\alpha}(x, y)=0$.

Part (3): It follows from $(4.3)^{\prime}$ that

$$
\begin{aligned}
\nabla_{x} \varphi_{\alpha}(0, y) & =y+\frac{1}{\alpha}\left[(-\alpha y)_{+}-\alpha y_{+}\right] \\
& =y+\left[(-y)_{+}-y_{+}\right] \\
& =y+\left[-y_{-}-y_{+}\right] \\
& =0
\end{aligned}
$$

where the second equality follows from $\alpha>0$ and (2.3); the third equality follows from $(-y)_{+}=$ $-y_{-}$; and the last equality follows from (2.4).

Part (4): Using a similar way as in Part (3), we obtain

$$
\begin{aligned}
\nabla_{y} \varphi_{\alpha}(0, y) & =0+\frac{1}{\alpha}\left[y_{+}-y-\alpha(-\alpha y)_{+}\right] \\
& =\frac{1}{\alpha}\left[-y_{-}+\alpha^{2} y_{-}\right] \\
& =\frac{1}{\alpha}\left(\alpha^{2}-1\right) y_{-} .
\end{aligned}
$$

The desired result follows immediately from $y_{-} \in(-K)$ by $(2.3)$, and $\alpha>1$ or $0<\alpha<1$.

In the setting of NCP, from Lemma 2.2 in Facchinei and Kanzow (1997) we know that $\nabla \varphi_{\alpha}(x, y) \circ \nabla_{y} \varphi_{\alpha}(x, y) \geq 0$. However, the following example shows that this statement can not be generalized to the setting of SCCP.

Consider the 4-dimensional second-order cone $\Lambda_{+}^{4}$. Let $x=(2,1,1,1)^{T} \in \Lambda_{+}^{4}$ and $y=$ $(2,1,1,0)^{T} \in \Lambda_{+}^{4}$ and $\alpha=2$. Then direct calculation yields

$$
\begin{aligned}
& x-2 y=\left(\begin{array}{c}
-2 \\
-1 \\
-1 \\
1
\end{array}\right) \in\left(-\Lambda_{+}^{4}\right), \\
& y-2 x=\left(\begin{array}{c}
-2 \\
-1 \\
-1 \\
-2
\end{array}\right)=\frac{1}{2}(-2+\sqrt{6})\left(\begin{array}{c}
1 \\
\frac{-1}{\sqrt{6}} \\
\frac{-1}{\sqrt{6}} \\
\frac{-2}{\sqrt{6}}
\end{array}\right)+\frac{1}{2}(-2-\sqrt{6})\left(\begin{array}{c}
1 \\
\frac{1}{\sqrt{6}} \\
\frac{1}{\sqrt{6}} \\
\frac{2}{\sqrt{6}}
\end{array}\right) .
\end{aligned}
$$

Furthermore, modelling on the proof of Part (2) in Proposition 4.4 we compute

$$
\begin{aligned}
\left(\alpha \nabla_{x} \varphi_{\alpha}\right) \circ\left(\alpha \nabla_{y} \varphi_{\alpha}\right) & =\alpha^{2} \nabla_{x} \varphi_{\alpha} \circ \nabla_{y} \varphi_{\alpha} \\
& =(x-\alpha y)_{-} \circ(y-\alpha x)_{-}+\alpha^{2}(x-\alpha y)_{+} \circ(y-\alpha x)_{+} \\
& =\left(\begin{array}{c}
-2 \\
-1 \\
-1 \\
1
\end{array}\right) \circ \frac{1}{2}(-2-\sqrt{6})\left(\begin{array}{c}
1 \\
\frac{1}{\sqrt{6}} \\
\frac{1}{\sqrt{6}} \\
\frac{2}{\sqrt{6}}
\end{array}\right)+0
\end{aligned}
$$




$$
=\frac{2+\sqrt{6}}{2 \sqrt{6}}\left(\begin{array}{c}
2 \sqrt{6} \\
2+\sqrt{6} \\
2+\sqrt{6} \\
4-\sqrt{6}
\end{array}\right) .
$$

Clearly, $\nabla_{x} \varphi_{\alpha} \circ \nabla_{y} \varphi_{\alpha} \notin \Lambda_{+}^{4}$. The desired claim follows.

In view of Theorem 4.3 and Proposition 4.4, we can derive a further equivalence result for the complementarity condition of problem (1.1).

Theorem 4.5 Let $\varphi_{\alpha}: \mathcal{J} \times \mathcal{J} \rightarrow R$ be given by (4.1). Then, the following statements are equivalent:

(a) $x \in K, \quad y \in K$, and $x \circ y=0$.

(b) $\nabla_{x} \varphi_{\alpha}(x, y)+\nabla_{y} \varphi_{\alpha}(x, y)=0$.

Proof. " $(a) \Rightarrow(b)$ " It is obvious from Theorem 4.3.

"(b) $\Rightarrow(a) "$ Given (b). Suppose on the contrary that $\nabla_{x} \varphi_{\alpha}(x, y) \neq 0$. From (b) and Proposition 4.4 (1), we have

$$
\begin{aligned}
0 & =\left\langle\nabla_{x} \varphi_{\alpha}(x, y), \nabla_{x} \varphi_{\alpha}(x, y)\right\rangle+\left\langle\nabla_{x} \varphi_{\alpha}(x, y), \nabla_{y} \varphi_{\alpha}(x, y)\right\rangle \\
& \geq\left\langle\nabla_{x} \varphi_{\alpha}(x, y), \nabla_{x} \varphi_{\alpha}(x, y)\right\rangle+0 \\
& >0
\end{aligned}
$$

a contradiction. So, $\nabla_{x} \varphi_{\alpha}(x, y)=0$, and hence $\nabla_{y} \varphi_{\alpha}(x, y)=0$.

Define the function $L(x, y):=\nabla_{x} \varphi_{\alpha}(x, y)+\nabla_{y} \varphi_{\alpha}(x, y)$. Then from Theorem 4.5 we know that $L(x, y)$ is a nonsmooth $C$-function for SCCP. Letting $L(x, y)$ equal zero and replacing $y$ by $F(x)$ with $L C^{1}$ property (i.e., $F$ is continuously differentiable and the Jacobian of $F$ is locally Lipschitz), by a simple calculation and Proposition 15 of Sun and Sun (2008), we obtain a strongly semismooth equation:

$$
\left[(x-\alpha F(x))_{+}-\left(x-\frac{1}{\alpha} F(x)\right)_{+}\right]+\left[(F(x)-\alpha x)_{+}-\left(F(x)-\frac{1}{\alpha} x\right)_{+}\right]=0
$$

for $\alpha \in(0,1)$ or $\alpha \in(1, \infty)$. It is an equivalent reformulation of problem (1.1), and has elegant symmetry with respect to $\alpha, x$ and $F(x)$. By using this equation we can design some iterative methods of the form

$$
x^{n e w}:=x-\left\{\left[(x-\alpha F(x))_{+}-\left(x-\frac{1}{\alpha} F(x)\right)_{+}\right]+\left[(F(x)-\alpha x)_{+}-\left(F(x)-\frac{1}{\alpha} x\right)_{+}\right]\right\},
$$

where $\alpha \in(0,1)$ or $\alpha \in(1, \infty)$ is some suitably chosen stepsize. Generally, such methods are first-order methods and may be appropriate for solving large-scale problems.

\section{$5 \quad$ Unconstrained Stationary Points}

In this section, for simplicity we always assume that $\alpha>1$ is arbitrary but fixed. If $y$ is replaced by $F(x)$ in the function $\varphi_{\alpha}$, then we get a composed function

$$
M(x, \alpha):=\varphi_{\alpha}(x, F(x)), \quad x \in \mathcal{J} .
$$


From Theorem 4.1, we know that $M(x, \alpha) \geq 0$ for any $x \in \mathcal{J}$, and $x^{*}$ solves problem (1.1) if and only if $M\left(x^{*}, \alpha\right)=0$. In other words, $M(x, \alpha)$ is a merit function for problem (1.1). Based on this, we have an unconstrained reformulation of problem (1.1)

$$
\min _{x \in \mathcal{J}} M(x, \alpha),
$$

where $\alpha>1$ is a parameter. Of course, $\alpha$ may be treated as a variable in $(1, \infty)$ if necessary. In general, we are only able to find the stationary points of problem (5.1). An interesting question is the following: Under what assumptions is a stationary point of $M(x, \alpha)$ a solution of problem (1.1)? In this section, we address the issue by giving a necessary and sufficient condition for a stationary point of $M(x, \alpha)$ to be a global solution of problem (5.1), and hence a solution of problem (1.1). We also show that this new condition easily allows us to establish some sufficient conditions.

We suppose in this section that $F$ is continuously differentiable at any $x \in \mathcal{J}$. In this case, the function $M(x, \alpha)$ is also continuously differentiable and its gradient at $x \in \mathcal{J}$ can be written as

$$
\nabla M(x, \alpha)=\nabla_{x} \varphi_{\alpha}(x, F(x))+F^{\prime}(x)^{T} \nabla_{y} \varphi_{\alpha}(x, F(x)) .
$$

From Theorems 4.1 and 4.3 , we readily derive the following theorem.

Theorem 5.1 Let $F$ be continuously differentiable and $x^{*} \in \mathcal{J}$ be a stationary point of $M(x, \alpha)$. Then, $x^{*}$ is a solution of problem (1.1) if and only if

$$
\nabla_{y} \varphi_{\alpha}\left(x^{*}, F\left(x^{*}\right)\right)=0 .
$$

Proof. Assume that $x^{*}$ solves problem (1.1). It is immediate from Theorems 4.1 and 4.3 that $\nabla_{y} \varphi_{\alpha}\left(x^{*}, F\left(x^{*}\right)\right)=0$.

Conversely, suppose that $\nabla_{y} \varphi_{\alpha}\left(x^{*}, F\left(x^{*}\right)\right)=0$. Since $x^{*}$ is a stationary point of $M(x, \alpha)$, we get from (5.2):

$$
\nabla_{x} \varphi_{\alpha}\left(x^{*}, F\left(x^{*}\right)\right)+F^{\prime}\left(x^{*}\right)^{T} \nabla_{y} \varphi_{\alpha}\left(x^{*}, F\left(x^{*}\right)\right)=0 .
$$

Thus, $\nabla_{x} \varphi_{\alpha}\left(x^{*}, F\left(x^{*}\right)\right)=0$. Furthermore, we have

$$
\nabla \varphi_{\alpha}\left(x^{*}, F\left(x^{*}\right)\right)=\left(\begin{array}{c}
\nabla_{x} \varphi_{\alpha}\left(x^{*}, F\left(x^{*}\right)\right) \\
\nabla_{y} \varphi_{\alpha}\left(x^{*}, F\left(x^{*}\right)\right)
\end{array}\right)=0 .
$$

This implies by Theorem 4.3 that $x^{*}$ is a solution of problem (1.1).

As done in Facchinei and Kanzow (1997) for NCP, we next give the definition of a regular point with respect to the merit function $M(x, \alpha)$, which needs to apply the properties of cone of a point given by Section 2, and will be central in the subsequent analysis.

Definition 5.2 $A$ point $x^{*} \in \mathcal{J}$ is called a regular point with respect to the merit function $M(x, \alpha)$ if, for every nonzero vector $z \in \operatorname{ri}\left(\operatorname{Cone}\left(\nabla_{y} \varphi_{\alpha}\left(x^{*}, F\left(x^{*}\right)\right)\right)\right) \cap\left(\nabla_{x} \varphi_{\alpha}\left(x^{*}, F\left(x^{*}\right)\right)\right)^{*}$, there exists a nonzero vector

$$
\omega \in \operatorname{Cone}\left(\nabla_{y} \varphi_{\alpha}\left(x^{*}, F\left(x^{*}\right)\right)\right) \bigcap\left(\nabla_{x} \varphi_{\alpha}\left(x^{*}, F\left(x^{*}\right)\right)\right)^{*}
$$

such that

$$
\left\langle\omega, F^{\prime}\left(x^{*}\right)^{T} z\right\rangle>0,
$$

where $\operatorname{ri}(C)$ denotes the relative interior of a set $C$ in $\mathcal{J}$, and $\left(\nabla_{x} \varphi_{\alpha}\left(x^{*}, F\left(x^{*}\right)\right)\right)^{*}=\{z \in \mathcal{J}$ : $\left.\left\langle z, \nabla_{x} \varphi_{\alpha}\left(x^{*}, F\left(x^{*}\right)\right)\right\rangle \geq 0\right\}$ is the dual cone. 
When $\mathcal{J}=R^{n}$ and $K=R_{+}^{n}$, this definition is consistent with that of a regular point for NCP introduced by Facchinei and Kanzow (1997). It follows from Lemma 2.2 in Facchinei and Kanzow (1997) that

$$
\begin{aligned}
& \operatorname{Cone}\left(\nabla_{y} \varphi_{\alpha}\left(x^{*}, F\left(x^{*}\right)\right)\right) \bigcap\left(\nabla_{x} \varphi_{\alpha}\left(x^{*}, F\left(x^{*}\right)\right)\right)^{*}=\operatorname{Cone}\left(\nabla_{y} \varphi_{\alpha}\left(x^{*}, F\left(x^{*}\right)\right)\right) \\
= & \left\{z \in R^{n}: z_{\ell}=0, z_{\wp} \geq 0, z_{\Im} \leq 0\right\},
\end{aligned}
$$

where the index sets $\ell, \wp, \Im$ are a partition of the index set $\{1, \cdots, n\}$ as in Facchinei and Kanzow (1997). Thus,

$$
\begin{aligned}
& \operatorname{ri}\left(\operatorname{Cone}\left(\nabla_{y} \varphi_{\alpha}\left(x^{*}, F\left(x^{*}\right)\right)\right)\right) \bigcap\left(\nabla_{x} \varphi_{\alpha}\left(x^{*}, F\left(x^{*}\right)\right)\right)^{*}=\operatorname{ri}\left(\operatorname{Cone}\left(\nabla_{y} \varphi_{\alpha}\left(x^{*}, F\left(x^{*}\right)\right)\right)\right) \\
= & \left\{z \in R^{n}: z_{\ell}=0, z_{\wp}>0, z_{\Im}<0\right\} .
\end{aligned}
$$

These conditions are the same as in Definition 3.1 of Facchinei and Kanzow (1997). So, ours is a generalization of the previous one.

In the context of second-order cone $\Lambda_{+}^{n}$, the set $\operatorname{Cone}\left(\nabla_{y} \varphi_{\alpha}\left(x^{*}, F\left(x^{*}\right)\right)\right) \cap\left(\nabla_{x} \varphi_{\alpha}\left(x^{*}, F\left(x^{*}\right)\right)\right)^{*}$ can be specified as follows (we partition the cases with respect to the signs of the eigenvalues of $\nabla_{y} \varphi_{\alpha}\left(x^{*}, F\left(x^{*}\right)\right)$, without loss of generality, $\left.\lambda_{1} \geq \lambda_{2}\right)$ :

Case 1:

$$
\lambda\left(\nabla_{y} \varphi_{\alpha}\left(x^{*}, F\left(x^{*}\right)\right)\right) \in\left\{\left(\begin{array}{c}
- \\
-
\end{array}\right),\left(\begin{array}{c}
+ \\
+
\end{array}\right)\right\} \text {. Then } \nabla_{y} \varphi_{\alpha}\left(x^{*}, F\left(x^{*}\right)\right) \in \operatorname{int} \Lambda_{+}^{n} \bigcup\left(-\operatorname{int} \Lambda_{+}^{n}\right) .
$$

Without loss of generality, let $\nabla_{y} \varphi_{\alpha}\left(x^{*}, F\left(x^{*}\right)\right) \in \operatorname{int} \Lambda_{+}^{n}$. By $(4.3)^{\prime}$,

$$
\nabla_{y} \varphi_{\alpha}\left(x^{*}, F\left(x^{*}\right)\right)=\frac{1}{\alpha}\left(\alpha x^{*}-F\left(x^{*}\right)\right)_{+}-\left(x^{*}-\alpha F\left(x^{*}\right)\right)_{+} .
$$

Thus

$$
\frac{1}{\alpha}\left(\alpha x^{*}-F\left(x^{*}\right)\right)_{+}=\nabla_{y} \varphi_{\alpha}\left(x^{*}, F\left(x^{*}\right)\right)+\left(x^{*}-\alpha F\left(x^{*}\right)\right)_{+} \in \operatorname{int} \Lambda_{+}^{n} .
$$

This implies $\alpha x^{*}-F\left(x^{*}\right) \in \operatorname{int} \Lambda_{+}^{n}$. Hence $\left(\alpha x^{*}-F\left(x^{*}\right)\right)_{-}=\left(F\left(x^{*}\right)-\alpha x^{*}\right)_{+}=0$. By $(4.3)^{\prime}$,

$$
\nabla_{x} \varphi_{\alpha}\left(x^{*}, F\left(x^{*}\right)\right)=\frac{1}{\alpha}\left(\alpha F\left(x^{*}\right)-x^{*}\right)_{+}-\left(F\left(x^{*}\right)-\alpha x^{*}\right)_{+}=\frac{1}{\alpha}\left(\alpha F\left(x^{*}\right)-x^{*}\right)_{+} \in \Lambda_{+}^{n} .
$$

Then $\Lambda_{+}^{n} \subseteq\left(\nabla_{x} \varphi_{\alpha}\left(x^{*}, F\left(x^{*}\right)\right)\right)^{*}$. Also, in this case, we have $\operatorname{Cone}\left(\nabla_{y} \varphi_{\alpha}\left(x^{*}, F\left(x^{*}\right)\right)\right) \subseteq \Lambda_{+}^{n}$. So, we obtain $\operatorname{Cone}\left(\nabla_{y} \varphi_{\alpha}\left(x^{*}, F\left(x^{*}\right)\right)\right) \cap\left(\nabla_{x} \varphi_{\alpha}\left(x^{*}, F\left(x^{*}\right)\right)\right)^{*}=\operatorname{Cone}\left(\nabla_{y} \varphi_{\alpha}\left(x^{*}, F\left(x^{*}\right)\right)\right)$.

$\underline{\text { Case 2: }}$

$$
\lambda\left(\nabla_{y} \varphi_{\alpha}\left(x^{*}, F\left(x^{*}\right)\right)\right) \in\left\{\left(\begin{array}{c}
0 \\
-
\end{array}\right),\left(\begin{array}{c}
0 \\
0
\end{array}\right),\left(\begin{array}{c}
+ \\
0
\end{array}\right)\right\} \text {. Then } \nabla_{y} \varphi_{\alpha}\left(x^{*}, F\left(x^{*}\right)\right) \in \partial \Lambda_{+}^{n} \bigcup\left(-\partial \Lambda_{+}^{n}\right) .
$$

Clearly, Cone $\left(\nabla_{y} \varphi_{\alpha}\left(x^{*}, F\left(x^{*}\right)\right)\right)$ is either a closed half-line, $\left\{\delta \nabla_{y} \varphi_{\alpha}\left(x^{*}, F\left(x^{*}\right)\right): \delta \geq 0\right\}$, if $\nabla_{y} \varphi_{\alpha}\left(x^{*}, F\left(x^{*}\right)\right) \neq 0$ or a singleton, $\{0\}$, if $\nabla_{y} \varphi_{\alpha}\left(x^{*}, F\left(x^{*}\right)\right)=0$. Therefore, by Proposition 4.4, part (1), we obtain that $\operatorname{Cone}\left(\nabla_{y} \varphi_{\alpha}\left(x^{*}, F\left(x^{*}\right)\right)\right) \cap\left(\nabla_{x} \varphi_{\alpha}\left(x^{*}, F\left(x^{*}\right)\right)\right)^{*}=\operatorname{Cone}\left(\nabla_{y} \varphi_{\alpha}\left(x^{*}, F\left(x^{*}\right)\right)\right)$.

$\underline{\text { Case 3: }}$

$$
\lambda\left(\nabla_{y} \varphi_{\alpha}\left(x^{*}, F\left(x^{*}\right)\right)\right) \in\left\{\left(\begin{array}{c}
+ \\
-
\end{array}\right)\right\} \text {. Then we have } \nabla_{y} \varphi_{\alpha}\left(x^{*}, F\left(x^{*}\right)\right) \notin \Lambda_{+}^{n} \bigcup\left(-\Lambda_{+}^{n}\right) .
$$


We apply a spectral decomposition and write

$$
\nabla_{y} \varphi_{\alpha}\left(x^{*}, F\left(x^{*}\right)\right)=\lambda_{1} e_{1}+\lambda_{2} e_{2},
$$

where two eigenvalues $\lambda_{1}>0>\lambda_{2}$ are unique and $\left\{e_{1}, e_{2}\right\}$ is the corresponding unique Jordan frame. It is easy to see that

$$
\operatorname{Cone}\left(\nabla_{y} \varphi_{\alpha}\left(x^{*}, F\left(x^{*}\right)\right)\right)=\left\{\theta_{1} e_{1}-\theta_{2} e_{2}: \theta_{1}, \theta_{2} \geq 0\right\} .
$$

Note that

$$
\left\langle\nabla_{y} \varphi_{\alpha}\left(x^{*}, F\left(x^{*}\right)\right), \nabla_{x} \varphi_{\alpha}\left(x^{*}, F\left(x^{*}\right)\right)\right\rangle=\lambda_{1}\left\langle e_{1}, \nabla_{y} \varphi_{\alpha}\left(x^{*}, F\left(x^{*}\right)\right)\right\rangle+\lambda_{2}\left\langle e_{2}, \nabla_{x} \varphi_{\alpha}\left(x^{*}, F\left(x^{*}\right)\right)\right\rangle \geq 0 .
$$

We need only to consider the following three subcases:

Subcase 3.1: $\left\langle\nabla_{x} \varphi_{\alpha}\left(x^{*}, F\left(x^{*}\right)\right), e_{1}\right\rangle \geq 0$ and $\left\langle\nabla_{x} \varphi_{\alpha}\left(x^{*}, F\left(x^{*}\right)\right), e_{2}\right\rangle \leq 0$. Clearly, for any $\theta_{1}, \theta_{2} \geq 0$ we have $\left\langle\nabla_{x} \varphi_{\alpha}\left(x^{*}, F\left(x^{*}\right)\right), \theta_{1} e_{1}-\theta_{2} e_{2}\right\rangle \geq 0$. Thus, we have

$$
\operatorname{Cone}\left(\nabla_{y} \varphi_{\alpha}\left(x^{*}, F\left(x^{*}\right)\right)\right) \bigcap\left(\nabla_{x} \varphi_{\alpha}\left(x^{*}, F\left(x^{*}\right)\right)\right)^{*}=\operatorname{Cone}\left(\nabla_{y} \varphi_{\alpha}\left(x^{*}, F\left(x^{*}\right)\right)\right) \text {. }
$$

Subcase 3.2: $\left\langle\nabla_{x} \varphi_{\alpha}\left(x^{*}, F\left(x^{*}\right)\right), e_{1}\right\rangle>0$ and $\left\langle\nabla_{x} \varphi_{\alpha}\left(x^{*}, F\left(x^{*}\right)\right), e_{2}\right\rangle>0$. Clearly, letting

$$
\lambda_{0}:=\frac{\left\langle\nabla_{x} \varphi_{\alpha}\left(x^{*}, F\left(x^{*}\right)\right), e_{1}\right\rangle}{\left\langle\nabla_{x} \varphi_{\alpha}\left(x^{*}, F\left(x^{*}\right)\right), e_{2}\right\rangle}
$$

we easily verify that $\lambda_{0}>0$ and $\left\langle\nabla_{x} \varphi_{\alpha}\left(x^{*}, F\left(x^{*}\right)\right), e_{1}-\lambda_{0} e_{2}\right\rangle=0$. Thus, we obtain that in this subcase

$$
\operatorname{Cone}\left(\nabla_{y} \varphi_{\alpha}\left(x^{*}, F\left(x^{*}\right)\right)\right) \bigcap\left(\nabla_{x} \varphi_{\alpha}\left(x^{*}, F\left(x^{*}\right)\right)\right)^{*}=\left\{\theta_{1} e_{1}+\theta_{2}\left(e_{1}-\lambda_{0} e_{2}\right): \theta_{1}, \theta_{2} \geq 0\right\} .
$$

Subcase 3.3: $\left\langle\nabla_{x} \varphi_{\alpha}\left(x^{*}, F\left(x^{*}\right)\right), e_{1}\right\rangle<0$ and $\left\langle\nabla_{x} \varphi_{\alpha}\left(x^{*}, F\left(x^{*}\right)\right), e_{2}\right\rangle<0$. Similar to Subcase 3.2 , letting $\lambda_{0}$ be specified by (5.4), we obtain that $\lambda_{0}>0$ and

$$
\operatorname{Cone}\left(\nabla_{y} \varphi_{\alpha}\left(x^{*}, F\left(x^{*}\right)\right)\right) \bigcap\left(\nabla_{x} \varphi_{\alpha}\left(x^{*}, F\left(x^{*}\right)\right)\right)^{*}=\left\{\theta_{1} e_{2}+\theta_{2}\left(e_{1}-\lambda_{0} e_{2}\right): \theta_{1}, \theta_{2} \geq 0\right\} .
$$

Furthermore, for the multiple SOCs $K_{+}^{n} \subset R^{n}$ as $K^{n}:=\Lambda^{n_{1}} \times \Lambda^{n_{2}} \times \cdots \times \Lambda^{n_{m}}$ and $K_{+}^{n}:=$ $\Lambda_{+}^{n_{1}} \times \Lambda_{+}^{n_{2}} \times \cdots \times \Lambda_{+}^{n_{m}}$ with $n=n_{1}+n_{2}+\cdots+n_{m}$, we have

$$
x \circ F(x)=\left(x^{(1)} \circ F^{(1)}(x), x^{(2)} \circ F^{(2)}(x), \cdots, x^{(m)} \circ F^{(m)}(x)\right)^{T}, \quad\langle x, F(x)\rangle=\sum_{\nu=1}^{m}\left\langle x^{(\nu)}, F^{(\nu)}(x)\right\rangle
$$

where $x=\left(x^{(1)}, x^{(2)}, \cdots, x^{(m)}\right)^{T}$ and $F(x)=\left(F^{(1)}(x), F^{(2)}(x), \cdots, F^{(m)}(x)\right)^{T}$ with $x^{(\nu)} \in \Lambda^{n_{\nu}}$ and $F^{(\nu)}(x) \in \Lambda^{n_{\nu}}$ for $\nu \in\{1,2, \cdots, m\}$. The function $\varphi_{\alpha}(x, F(x))$ can be written as

$$
\varphi_{\alpha}(x, F(x))=\sum_{\nu=1}^{m} \varphi_{\alpha}^{(\nu)}\left(x^{(\nu)}, F^{(\nu)}(x)\right) .
$$

The gradients of $\varphi_{\alpha}$ become respectively

$$
\nabla_{x} \varphi_{\alpha}(x, F(x))=\left(\nabla_{x^{(1)}} \varphi_{\alpha}^{(1)}\left(x^{(1)}, F^{(1)}(x)\right), \cdots, \nabla_{x^{(m)}} \varphi_{\alpha}^{(m)}\left(x^{(m)}, F^{(m)}(x)\right)\right)^{T}
$$

and

$$
\nabla_{y} \varphi_{\alpha}(x, F(x))=\left(\nabla_{y^{(1)}} \varphi_{\alpha}^{(1)}\left(x^{(1)}, F^{(1)}(x)\right), \cdots, \nabla_{y^{(m)}} \varphi_{\alpha}^{(m)}\left(x^{(m)}, F^{(m)}(x)\right)\right)^{T} .
$$


Thus we have

$\operatorname{Cone}\left(\nabla_{y} \varphi_{\alpha}\left(x^{*}, F\left(x^{*}\right)\right)\right)=\operatorname{Cone}\left(\nabla_{y^{(1)}} \varphi_{\alpha}\left(x^{*(1)}, F^{(1)}\left(x^{*}\right)\right)\right) \times \cdots \times \operatorname{Cone}\left(\nabla_{y^{(m)}} \varphi_{\alpha}\left(x^{*(m)}, F^{(m)}\left(x^{*}\right)\right)\right)$, and

$$
\left(\nabla_{x} \varphi_{\alpha}\left(x^{*}, F\left(x^{*}\right)\right)\right)^{*}=\left(\nabla_{x^{(1)}} \varphi_{\alpha}\left(x^{*(1)}, F^{(1)}\left(x^{*}\right)\right)\right)^{*} \times \cdots \times\left(\nabla_{x^{(m)}} \varphi_{\alpha}\left(x^{*(m)}, F^{(m)}\left(x^{*}\right)\right)\right)^{*} .
$$

Therefore, we obtain that

$$
\begin{aligned}
& \operatorname{Cone}\left(\nabla_{y} \varphi_{\alpha}\left(x^{*}, F\left(x^{*}\right)\right)\right) \bigcap\left(\nabla_{x} \varphi_{\alpha}\left(x^{*}, F\left(x^{*}\right)\right)\right)^{*} \\
= & \operatorname{Cone}\left(\nabla_{y^{(1)}} \varphi_{\alpha}\left(x^{*(1)}, F^{(1)}\left(x^{*}\right)\right)\right) \bigcap\left(\nabla_{x^{(1)}} \varphi_{\alpha}\left(x^{*(1)}, F^{(1)}\left(x^{*}\right)\right)\right)^{*} \\
& \times \cdots \times \operatorname{Cone}\left(\nabla_{y^{(m)}} \varphi_{\alpha}\left(x^{*(m)}, F^{(m)}\left(x^{*}\right)\right)\right) \bigcap\left(\nabla_{x^{(m)}} \varphi_{\alpha}\left(x^{*(m)}, F^{(m)}\left(x^{*}\right)\right)\right)^{*} .
\end{aligned}
$$

From the same arguments as in the single SOC (Cases 1-3), we easily obtain that for $\nu \in$ $\{1,2, \cdots, m\}$, the set $\operatorname{Cone}\left(\nabla_{y^{(\nu)}} \varphi_{\alpha}\left(x^{*(\nu)}, F^{(\nu)}\left(x^{*}\right)\right)\right) \cap\left(\nabla_{x^{(\nu)}} \varphi_{\alpha}\left(x^{*(\nu)}, F^{(\nu)}\left(x^{*}\right)\right)\right)^{*}$ has the similar form corresponding to the signs of eigenvalues of $\nabla_{y^{(\nu)}} \varphi_{\alpha}\left(x^{*(\nu)}, F^{(\nu)}\left(x^{*}\right)\right)$ in $\Lambda^{n_{\nu}}$.

We are now in position to prove the main result of this section.

Theorem 5.3 Let $F$ be continuously differentiable and $x^{*} \in \mathcal{J}$ be a stationary point of $M(x, \alpha)$. Then, $x^{*}$ solves problem (1.1) if and only if $x^{*}$ is regular.

Proof. Suppose that $x^{*} \in \mathcal{J}$ is a solution of problem (1.1). Then by Theorem 5.1, we have Cone $\left(\nabla_{y} \varphi_{\alpha}\left(x^{*}, F\left(x^{*}\right)\right)\right)=\{0\}$. Therefore, there is no nonzero vector satisfying $z \in$ $\operatorname{ri}\left(\operatorname{Cone}\left(\nabla_{y} \varphi_{\alpha}\left(x^{*}, F\left(x^{*}\right)\right)\right)\right) \cap\left(\nabla_{x} \varphi_{\alpha}\left(x^{*}, F\left(x^{*}\right)\right)\right)^{*}$.

Conversely, we observe that (5.2) holds since $x^{*}$ is a stationary point of $M(x, \alpha)$. So, for any $\omega \in \mathcal{J}$,

$$
\left\langle\omega, \nabla_{x} \varphi_{\alpha}\left(x^{*}, F\left(x^{*}\right)\right)+F^{\prime}\left(x^{*}\right)^{T} \nabla_{y} \varphi_{\alpha}\left(x^{*}, F\left(x^{*}\right)\right\rangle=0 .\right.
$$

Suppose that $x^{*}$ is not a solution of problem (1.1). Then by Theorem $5.1, \nabla_{y} \varphi_{\alpha}\left(x^{*}, F\left(x^{*}\right)\right)$ is a nonzero vector. Setting $z:=\nabla_{y} \varphi_{\alpha}\left(x^{*}, F\left(x^{*}\right)\right)$, by Propositions 2.4 and 4.4, we have $z \in$ $\operatorname{ri}\left(\right.$ Cone $\left.\left(\nabla_{y} \varphi_{\alpha}\left(x^{*}, F\left(x^{*}\right)\right)\right)\right) \cap\left(\nabla_{x} \varphi_{\alpha}\left(x^{*}, F\left(x^{*}\right)\right)\right)^{*}$. Since $x^{*}$ is regular, by Definition 5.2 , there is a nonzero vector

$$
\omega^{*} \in \operatorname{Cone}\left(\nabla_{y} \varphi_{\alpha}\left(x^{*}, F\left(x^{*}\right)\right)\right) \bigcap\left(\nabla_{x} \varphi_{\alpha}\left(x^{*}, F\left(x^{*}\right)\right)\right)^{*}
$$

such that

$$
\left\langle\omega^{*}, F^{\prime}\left(x^{*}\right)^{T} \nabla_{y} \varphi_{\alpha}\left(x^{*}, F\left(x^{*}\right)\right\rangle=\left\langle\omega^{*}, F^{\prime}\left(x^{*}\right)^{T} z\right\rangle>0 .\right.
$$

In addition, from the definition of dual cone, we obtain

$$
\left\langle\omega^{*}, \nabla_{x} \varphi_{\alpha}\left(x^{*}, F\left(x^{*}\right)\right)\right\rangle \geq 0 .
$$

Thus,

$$
\begin{aligned}
& \left\langle\omega^{*}, \nabla_{x} \varphi_{\alpha}\left(x^{*}, F\left(x^{*}\right)\right)+F^{\prime}\left(x^{*}\right)^{T} \nabla_{y} \varphi_{\alpha}\left(x^{*}, F\left(x^{*}\right)\right\rangle\right. \\
= & \left\langle\omega^{*}, \nabla_{x} \varphi_{\alpha}\left(x^{*}, F\left(x^{*}\right)\right)\right\rangle+\left\langle\omega^{*}, F^{\prime}\left(x^{*}\right)^{T} \nabla_{y} \varphi_{\alpha}\left(x^{*}, F\left(x^{*}\right)\right\rangle\right. \\
> & 0 .
\end{aligned}
$$

This is a contradiction to (5.5), and hence $x^{*}$ solves problem (1.1). 
We end this section with the following corollary which gives a sufficient condition for a stationary point to be a solution of problem (1.1).

Corollary 5.4 Let $F$ be continuously differentiable and $x^{*} \in \mathcal{J}$ be a stationary point of $M(x, \alpha)$. If the Jacobian $F^{\prime}\left(x^{*}\right)$ is positive definite, then $x^{*}$ is a regular solution of problem (1.1).

Proof. In view of Theorem 5.3, it suffices to show that $x^{*}$ is regular. Let $z \in \mathcal{J}$ be any nonzero vector in the set $\operatorname{ri}\left(\operatorname{Cone}\left(\nabla_{y} \varphi_{\alpha}\left(x^{*}, F\left(x^{*}\right)\right)\right)\right) \cap\left(\nabla_{x} \varphi_{\alpha}\left(x^{*}, F\left(x^{*}\right)\right)\right)^{*}$, then by taking $\omega=z$, one has

$$
\omega \in \operatorname{Cone}\left(\nabla_{y} \varphi_{\alpha}\left(x^{*}, F\left(x^{*}\right)\right)\right) \bigcap\left(\nabla_{x} \varphi_{\alpha}\left(x^{*}, F\left(x^{*}\right)\right)\right)^{*}
$$

Since, by assumption, $F^{\prime}\left(x^{*}\right)$ and therefore also $F^{\prime}\left(x^{*}\right)^{T}$ is positive definite, we have

$$
\left\langle\omega, F^{\prime}\left(x^{*}\right)^{T} z\right\rangle=\left\langle z, F^{\prime}\left(x^{*}\right)^{T} z\right\rangle>0 .
$$

So, $x^{*}$ is a regular point.

In the setting of NCP, this corollary reduces to the result by Yamashita and Fukushima (1995). In next section we shall develop another sufficient condition which generalizes Corollary 5.4 .

\section{Cartesian P-property and Error Bound}

We first recall the structure theorems of a Euclidean Jordan algebra, i.e., Propositions III.4.4 and III.4.5, Theorem V.3.7 in Faraut and Korányi (1994). From these results we know that for a given Euclidean Jordan algebra $\mathcal{J}$ and the corresponding symmetric cone $K$, we have

$$
\mathcal{J}=\mathcal{J}_{1} \times \mathcal{J}_{2} \times \cdots \times \mathcal{J}_{m} \text { and } K=K_{1} \times K_{2} \times \cdots \times K_{m},
$$

where each $n_{\nu}$-dimensional space $\mathcal{J}_{\nu}$ is a simple Jordan algebra (which is not the direct sum of two Euclidean Jordan algebras) with the corresponding symmetric cone $K_{\nu}$, and $n=\sum_{\nu=1}^{m} n_{\nu}$. Moreover, for $x=\left(x^{(1)}, x^{(2)}, \cdots, x^{(m)}\right)^{T}$ and $y=\left(y^{(1)}, y^{(2)}, \cdots, y^{(m)}\right)^{T}$ in $\mathcal{J}$ with $x^{(\nu)}, y^{(\nu)} \in \mathcal{J}_{\nu}$, one also has

$$
x \circ y=\left(x^{(1)} \circ y^{(1)}, x^{(2)} \circ y^{(2)}, \cdots, x^{(m)} \circ y^{(m)}\right)^{T}
$$

and

$$
\langle x, y\rangle=\sum_{\nu=1}^{m}\left\langle x^{(\nu)}, y^{(\nu)}\right\rangle .
$$

Therefore, the complementarity condition of problem (1.1) is equivalent to

$$
x^{(\nu)} \in K_{\nu}, \quad y^{(\nu)} \in K_{\nu}, \quad x^{(\nu)} \circ y^{(\nu)}=0
$$

for each $\nu \in\{1,2, \cdots, m\}$. The functions $\Phi_{\alpha}(x, y)$ and $\varphi_{\alpha}(x, y)$ can be written as

$$
\Phi_{\alpha}(x, y)=\left(\Phi_{\alpha}^{(1)}\left(x^{(1)}, y^{(1)}\right), \Phi_{\alpha}^{(2)}\left(x^{(2)}, y^{(2)}\right), \cdots, \Phi_{\alpha}^{(m)}\left(x^{(m)}, y^{(m)}\right)\right)^{T}
$$

and

$$
\varphi_{\alpha}(x, y)=\sum_{\nu=1}^{m} \varphi_{\alpha}^{(\nu)}\left(x^{(\nu)}, y^{(\nu)}\right)=\sum_{\nu=1}^{m}\left\langle e^{(\nu)}, \Phi_{\alpha}^{(\nu)}\left(x^{(\nu)}, y^{(\nu)}\right)\right\rangle
$$


respectively. The gradients of $\varphi_{\alpha}$ with respect to $x$ and $y$ become respectively

$$
\nabla_{x} \varphi_{\alpha}(x, y)=\left(\nabla_{x^{(1)}} \varphi_{\alpha}^{(1)}\left(x^{(1)}, y^{(1)}\right), \cdots, \nabla_{x^{(m)}} \varphi_{\alpha}^{(m)}\left(x^{(m)}, y^{(m)}\right)\right)^{T}
$$

and

$$
\nabla_{y} \varphi_{\alpha}(x, y)=\left(\nabla_{y^{(1)}} \varphi_{\alpha}^{(1)}\left(x^{(1)}, y^{(1)}\right), \cdots, \nabla_{y^{(m)}} \varphi_{\alpha}^{(m)}\left(x^{(m)}, y^{(m)}\right)\right)^{T} .
$$

It is easy to verify that all the results in Sections 3 and 4 hold in the setting of Jordan algebra $\mathcal{J}_{\nu}$, such as the inequality

$$
\left\langle\nabla_{x^{(\nu)}} \varphi_{\alpha}^{(\nu)}\left(x^{(\nu)}, y^{(\nu)}\right), \nabla_{y^{(\nu)}} \varphi_{\alpha}^{(\nu)}\left(x^{(\nu)}, y^{(\nu)}\right)\right\rangle \geq 0
$$

holds for each $\nu \in\{1,2, \cdots, m\}$; see Proposition 4.4(1).

Motivated by $P$-properties on Cartesian products in $R^{n}$ established by Facchinei and Pang (2003) and Cartesian $P$-properties in the setting of $S^{n}$ developed by Chen and Qi (2006), we introduce the concept of Cartesian P-property for the nonlinear transformation $F$ in the setting of Jordan algebra.

Definition 6.1 For a mapping $F: \mathcal{J} \rightarrow \mathcal{J}$ with $\mathcal{J}$ given by the form of Cartesian products, we say that it has

(i) the Cartesian P-property if for any pair $x, y \in \mathcal{J}(x \neq y)$, there exists an index $\nu \in$ $\{1,2, \cdots, m\}$ such that

$$
\left\langle(x-y)^{(\nu)},(F(x)-F(y))^{(\nu)}\right\rangle>0 ;
$$

(ii) the uniform Cartesian P-property if for any pair $x, y \in \mathcal{J}(x \neq y)$, there exist an index $\nu \in\{1,2, \cdots, m\}$ and a positive scalar $\rho$ such that

$$
\left\langle(x-y)^{(\nu)},(F(x)-F(y))^{(\nu)}\right\rangle \geq \rho\|x-y\|^{2} ;
$$

(iii) the Cartesian $P_{0}$-property if for any pair $x, y \in \mathcal{J}(x \neq y)$, there exists an index $\nu \in\{1,2, \cdots, m\}$ such that

$$
x^{(\nu)} \neq y^{(\nu)} \text { and }\left\langle(x-y)^{(\nu)},(F(x)-F(y))^{(\nu)}\right\rangle \geq 0 .
$$

It is easy to observe that when $m=1$, the (uniform) Cartesian $(P) P_{0}$-property becomes the (strong) monotonicity of $F$, and when $m=n$ with $\mathcal{J}=R^{n}$, it becomes the $(P) P_{0}$-property in the context of NCP. Especially, when $F(x)$ is continuously differentiable, the Jacobian $F^{\prime}(x)$ and its adjoint operator $F^{\prime}(x)^{T}$ at $x \in \mathcal{J}$ have the Cartesian $P$-property, i.e., for any vector $z \in \mathcal{J}$, there is an index $\nu \in\{1,2, \cdots, m\}$ such that

$$
\left\langle z^{(\nu)},\left(F^{\prime}(x) z\right)^{(\nu)}\right\rangle \geq \rho\|z\|^{2} \text { and }\left\langle z^{(\nu)},\left(F^{\prime}(x)^{T} z\right)^{(\nu)}\right\rangle \geq \rho\|z\|^{2}
$$

if $F$ has the uniform Cartesian $P$-property on $\mathcal{J}$. Similarly, we can define the Cartesian $P_{0^{-}}$ property of $F^{\prime}(x)$ at $x \in \mathcal{J}$.

Tao and Gowda (2005) introduced the concept of uniform Jordan P-property in the setting of Jordan algebra, and showed that in this case, problem (1.1) has a nonempty and bounded solution set. From the related definitions, we readily verify the following one-way implication of the properties for nonlinear transformation $F$ :

Strong Monotonicity $\Rightarrow$ Uniform Cartesian P-property $\Rightarrow$ Uniform Jordan P-property. 
Our Cartesian P-property strengthens the uniform Jordan P-property, and allows us to attain globally unique solvability (GUS-property) of the problem and an error bound result which does not require $F$ to be Lipschitz continuous and can be regarded as an extension of the corresponding result for box-constrained variational inequality given by Theorem 1 in Solodov and Tseng (2000).

Theorem 6.2 Let $F: \mathcal{J} \rightarrow \mathcal{J}$ be a continuous function. If $F$ has the uniform Cartesian $P$-property on $\mathcal{J}$ with modulus $\rho>0$, then

(i) the problem (1.1) has a unique solution, say $x^{*}$;

(ii) moreover, for every $\alpha>\max \left\{1, \frac{1}{2 \rho}\right\}$,

$$
\left\|x-x^{*}\right\|^{2} \leq c \cdot g(x, F(x), \alpha), \quad \forall x \in K,
$$

where $c:=1 /\left(\alpha \rho-\frac{1}{2}\right)$ is a positive scalar, and $g(x, y, \alpha)$ is the regularized gap function given by (3.10).

Proof. (i) We only prove the uniqueness of the solution to problem (1.1), since the existence of the solution is obtained in Proposition 3.2 and Corollary 3.1 by Tao and Gowda (2005). Assume that problem (1.1) admits two solutions, say $x, y \in \mathcal{J}$ with $x \neq y$. Then by the assumptions and Definition 6.1, there exists an index $v \in\{1,2, \cdots, m\}$ such that

$$
\left\langle(x-y)^{(\nu)},(F(x)-F(y))^{(\nu)}\right\rangle>0 .
$$

On the other hand, direct computation yields

$$
\begin{aligned}
\left\langle(x-y)^{(\nu)},(F(x)-F(y))^{(\nu)}\right\rangle & =\left\langle x^{(\nu)}-y^{(\nu)}, F(x)^{(\nu)}-F(y)^{(\nu)}\right\rangle \\
& =-\left\langle x^{(\nu)}, F(y)^{(\nu)}\right\rangle-\left\langle y^{(\nu)}, F(x)^{(\nu)}\right\rangle \\
& \leq 0,
\end{aligned}
$$

where the second equality follows from $\left\langle x^{(\nu)}, F(x)^{(\nu)}\right\rangle=\left\langle y^{(\nu)}, F(y)^{(\nu)}\right\rangle=0$, because $x$ and $y$ solve problem (1.1); and the inequality follows from $x^{(\nu)}, y^{(\nu)}, F(x)^{(\nu)}$ and $F(y)^{(\nu)}$ belonging to $K_{\nu}$. This is a contradiction.

(ii) Since $F$ has the uniform Cartesian P-property on $\mathcal{J}$, for any fixed $x \in K$ there exists an index $\nu \in\{1,2, \cdots, m\}$ such that

$$
\begin{aligned}
\rho\left\|x-x^{*}\right\|^{2} & \leq\left\langle x^{(\nu)}-x^{*(\nu)}, F(x)^{(\nu)}-F\left(x^{*}\right)^{(\nu)}\right\rangle \\
& =\left\langle x^{(\nu)}-x^{*(\nu)}, F(x)^{(\nu)}\right\rangle-\left\langle x^{(\nu)}, F\left(x^{*}\right)^{(\nu)}\right\rangle+\left\langle x^{*(\nu)}, F\left(x^{*}\right)^{(\nu)}\right\rangle \\
& \leq\left\langle x^{(\nu)}-x^{*(\nu)}, F(x)^{(\nu)}\right\rangle,
\end{aligned}
$$

where the last inequality is due to $x^{(\nu)} \in K_{\nu}, F\left(x^{*}\right)^{(\nu)} \in K_{\nu}$, and $\left\langle x^{*(\nu)}, F\left(x^{*}\right)^{(\nu)}\right\rangle=0$.

For $g(x, y, \alpha)$ given by (3.10), by the structure of Cartesian products it can be written as

$$
g(x, F(x), \alpha)=\sum_{i=1}^{m} g^{(i)}\left(x^{(i)}, F(x)^{(i)}, \alpha\right)
$$

with $g^{(i)}\left(x^{(i)}, F(x)^{(i)}, \alpha\right) \geq 0$ for $x^{(i)} \in K_{i}, i=1,2, \cdots, m$, because $x \in K$ is a feasible solution of the closest point problem (3.3). Thus, from (6.4) we obtain that

$$
\begin{aligned}
g(x, F(x), \alpha) & \geq g^{(\nu)}\left(x^{(\nu)}, F(x)^{(\nu)}, \alpha\right) \\
& =\max _{z \in K_{\nu}}\left\{\left\langle\alpha F(x)^{(\nu)}, x^{(\nu)}-z\right\rangle-\frac{1}{2}\left\|x^{(\nu)}-z\right\|^{2}\right\} \\
& \geq\left\langle\alpha F(x)^{(\nu)}, x^{(\nu)}-x^{*(\nu)}\right\rangle-\frac{1}{2}\left\|x^{(\nu)}-x^{*(\nu)}\right\|^{2} \\
& \geq \alpha \rho\left\|x-x^{*}\right\|^{2}-\frac{1}{2}\left\|x-x^{*}\right\|^{2} \\
& =\left(\alpha \rho-\frac{1}{2}\right)\left\|x-x^{*}\right\|^{2}
\end{aligned}
$$


for $x \in K$. This completes the proof.

Now we discuss how the merit function $M(x, \alpha)$ provides a global error bound for problem (1.1) with the uniform Cartesian P-property.

Theorem 6.3 Suppose that $F$ has the uniform Cartesian P-property with modulus $\rho>0$ and is Lipschitz continuous with constant $L>0$. Then for any fixed $\alpha>1$, there exist two positive scalars $c_{1}=\frac{1}{(\alpha-1)(2+L)^{2}}$ and $c_{2}=\frac{\alpha(1+L)^{2}}{(\alpha-1) \rho^{2}}$ such that

$$
c_{1} \cdot M(x, \alpha) \leq\left\|x-x^{*}\right\|^{2} \leq c_{2} \cdot M(x, \alpha), \quad \forall x \in \mathcal{J},
$$

where $x^{*}$ is the unique solution of problem (1.1).

Proof. Applying Theorem 3.2 in Tseng, Yamashita and Fukushima (1996) (or Theorem 4.2 in Peng 1997), we immediately obtain for any fixed $\alpha>1$ that

$$
\frac{\alpha-1}{\alpha}\left\|R_{1}(x)\right\|^{2} \leq M(x, \alpha) \leq(\alpha-1)\left\|R_{1}(x)\right\|^{2}, \forall x \in \mathcal{J},
$$

where $R_{1}(x):=x-(x-F(x))_{+}$. So, under the given assumptions, it suffices to prove the inequality

$$
\frac{1}{2+L}\left\|R_{1}(x)\right\| \leq\left\|x-x^{*}\right\| \leq \frac{1+L}{\rho}\left\|R_{1}(x)\right\|, \forall x \in \mathcal{J} .
$$

In fact, by the definition of $R_{1}$, for every $x \in \mathcal{J}$ we have

$$
F(x)-R_{1}(x)=-(x-F(x))_{-} \in K, \quad x-R_{1}(x)=(x-F(x))_{+} \in K .
$$

Clearly, $\left(F(x)-R_{1}(x)\right) \circ\left(x-R_{1}(x)\right)=0$ and $\left\langle F(x)-R_{1}(x), x-R_{1}(x)\right\rangle=0$. Thus, for any $v=1,2, \cdots, m$, we obtain

$$
\begin{aligned}
0= & \left\langle\left[F(x)-R_{1}(x)\right]^{(\nu)},\left[x-R_{1}(x)\right]^{(\nu)}\right\rangle \\
\geq & \left\langle\left[F(x)-R_{1}(x)\right]^{(\nu)}-\left[F\left(x^{*}\right)-R_{1}\left(x^{*}\right)\right]^{(\nu)},\left[x-R_{1}(x)\right]^{(\nu)}-\left[x^{*}-R_{1}\left(x^{*}\right)\right]^{(\nu)}\right\rangle \\
= & \left\langle F(x)^{(\nu)}-F\left(x^{*}\right)^{(\nu)}-R_{1}(x)^{(\nu)}+R_{1}\left(x^{*}\right)^{(\nu)}, x^{(\nu)}-x^{*(\nu)}-R_{1}(x)^{(\nu)}+R_{1}\left(x^{*}\right)^{(\nu)}\right\rangle \\
\geq & \left\langle F(x)^{(\nu)}-F\left(x^{*}\right)^{(\nu)}, x^{(\nu)}-x^{*(\nu)}\right\rangle-\left\langle R_{1}(x)^{(\nu)}+R_{1}\left(x^{*}\right)^{(\nu)}, F(x)^{(\nu)}-F\left(x^{*}\right)^{(\nu)}\right. \\
& \left.+x^{(\nu)}-x^{*(\nu)}\right\rangle \\
\geq & \left\langle F(x)^{(\nu)}-F\left(x^{*}\right)^{(\nu)}, x^{(\nu)}-x^{*(\nu)}\right\rangle-\left\|R_{1}(x)\right\| \cdot(1+L)\left\|x-x^{*}\right\|,
\end{aligned}
$$

where the first inequality is due to $\left[F(x)-R_{1}(x)\right]^{(\nu)} \in K_{\nu},\left[x-R_{1}(x)\right]^{(\nu)} \in K_{\nu},\left[F\left(x^{*}\right)-\right.$ $\left.R_{1}\left(x^{*}\right)\right]^{(\nu)} \in K_{\nu},\left[x^{*}-R_{1}\left(x^{*}\right)\right]^{(\nu)} \in K_{\nu}$, and $\left\langle\left[F\left(x^{*}\right)-R_{1}\left(x^{*}\right)\right]^{(\nu)},\left[x^{*}-R_{1}\left(x^{*}\right)\right]^{(\nu)}\right\rangle=0$; the last inequality follows from $\|x\|=\sqrt{\sum_{\nu=1}^{m}\left\|x^{(\nu)}\right\|^{2}} \geq\left\|x^{(\nu)}\right\|$ and the Lipschitz continuity of $F$. Thus, we conclude from the above inequality and the uniform Cartesian $P$-property of $F$,

$$
\begin{aligned}
(1+L)\left\|R_{1}(x)\right\|\left\|x-x^{*}\right\| & \geq \max _{1 \leq \nu \leq m}\left\langle F(x)^{(\nu)}-F\left(x^{*}\right)^{(\nu)}, x^{(\nu)}-x^{*(\nu)}\right\rangle \\
& \geq \rho\left\|x-x^{*}\right\|^{2} .
\end{aligned}
$$

This leads to the right-hand side of inequality (6.7). 
Note that $\left\|y_{+}-z_{+}\right\| \leq\|y-z\|$ for any $y, z \in \mathcal{J}$ by the property of projection. From the Lipschitz continuity of $F$, we obtain by direct manipulation that

$$
\begin{aligned}
\left\|R_{1}(x)\right\| & =\left\|\left[x-(x-F(x))_{+}\right]-\left[x^{*}-\left(x^{*}-F\left(x^{*}\right)\right)_{+}\right]\right\| \\
& =\left\|\left[x-x^{*}\right]-\left[(x-F(x))_{+}-\left(x^{*}-F\left(x^{*}\right)\right)_{+}\right]\right\| \\
& \leq\left\|x-x^{*}\right\|+\left\|(x-F(x))_{+}-\left(x^{*}-F\left(x^{*}\right)\right)_{+}\right\| \\
& \leq\left\|x-x^{*}\right\|+\left\|(x-F(x))-\left(x^{*}-F\left(x^{*}\right)\right)\right\| \\
& \leq 2\left\|x-x^{*}\right\|+\left\|F(x)-F\left(x^{*}\right)\right\| \\
& \leq(2+L)\left\|x-x^{*}\right\| .
\end{aligned}
$$

This means that the left-hand side of inequality (6.7) holds.

Combining (6.6) and (6.7), and taking $c_{1}=\frac{1}{(\alpha-1)(2+L)^{2}}$ and $c_{2}=\frac{\alpha(1+L)^{2}}{(\alpha-1) \rho^{2}}$, the desired conclusion (6.5) follows.

When $m=n$ with $\mathcal{J}=R^{n}$, the error bound result given by (6.7) becomes the corresponding one for NCP presented by Chen and Harker (1997). So, ours is a generalization of their result.

At last, we show that the uniform Cartesian P-property of $F$ is also a sufficient condition for the stationary point to be a solution of the problem.

Corollary 6.4 Let $F: \mathcal{J} \rightarrow \mathcal{J}$ be a continuously differentiable function, and $x^{*} \in \mathcal{J}$ be a stationary point of $M(x, \alpha)$. If $F$ has the uniform Cartesian P-property on $\mathcal{J}$, then $x^{*}$ is a regular solution of problem (1.1).

Proof. In view of Theorem 5.3, we only prove the regularity of $x^{*}$. Let $z \in \mathcal{J}$ be any nonzero vector in the set $\operatorname{ri}\left(\operatorname{Cone}\left(\nabla_{y} \varphi_{\alpha}\left(x^{*}, F\left(x^{*}\right)\right)\right)\right) \cap\left(\nabla_{x} \varphi_{\alpha}\left(x^{*}, F\left(x^{*}\right)\right)\right)^{*}$, then by the assumptions, there exists an index $\nu \in\{1,2, \cdots, m\}$ such that

$$
\left\langle z^{(\nu)},\left(F^{\prime}\left(x^{*}\right)^{T} z\right)^{(\nu)}\right\rangle>0 .
$$

Let $\omega \in \mathcal{J}$ be the vector whose blocks are all 0 except for its $\nu$ th block which is equal to $z^{(\nu)}$. Then, by (6.2) we readily show that $\omega$ satisfies all conditions in the definition of regularity. Hence, from (6.8) we obtain that

$$
\left\langle\omega, F^{\prime}\left(x^{*}\right)^{T} z\right\rangle=\left\langle z^{(\nu)},\left(F^{\prime}\left(x^{*}\right)^{T} z\right)^{(\nu)}\right\rangle>0,
$$

i.e., $x^{*}$ is a regular point. This completes the proof.

Based on the proofs of Corollaries 5.4 and 6.4, we assert that our regularity for a point is a weaker condition than the Cartesian P-property and the positive definiteness of the Jacobian $F^{\prime}(x)$ at this point.

\section{$7 \quad$ Final Remarks}

In this paper, we have introduced the vector-valued implicit Lagrangian for SCCP, and showed that it is a C-function and possesses several interesting properties. Then, we have developed the real-valued implicit Lagrangian and the related merit function for SCCP. Based on this merit function, we have presented a necessary and sufficient condition for a stationary point to be 
a solution of the problem (1.1), and established a global error bound for SCCP with uniform Cartesian P-property.

Finally, we should point out that the regularity of a point given in Section 5 is weaker than the Cartesian P-property, and provides a characterization of an important class of tractable nonmonotone SCCPs. However, we do not know the relationship between the regularity of a point and the various P-properties introduced by Gowda et al (2004, 2005). So, one future research topic motivated by our work is to investigate this relationship. Moreover, in this paper we do not address any numerical method for solving the symmetric cone complementarity problem based on the implicit Lagrangian. We leave this also as a future research topic.

Acknowledgments The authors are grateful to the anonymous referees for their very helpful and valuable comments. The work was partly supported by the National Natural Science Foundation of China (10671010, 70471002), and a Discovery Grant from NSERC.

\section{References}

Auchmuty, G. (1989), Variational principles for variational inequalities, Numerical Functional Analysis and Optimization 10, 863-874.

Chen, B. and P.T. Harker (1997), Smooth approximations to nonlinear complementarity problems, SIAM Journal on Optimization 7, 403-422.

Chen, X. and H. Qi (2006), Cartesian P-property and its applications to the semidefinite linear complementarity problem, Mathematical Programming 106, 177-201.

Facchinei F. and C. Kanzow (1997), On unconstrained and constrained stationary points of the Implicit Lagrangian, Journal of Optimization Theory and Applications 92, 99-115.

Facchinei, F. and J.-S. Pang (2003), Finite-Dimensional Variational Inequalities and Complementarity Problems Volume I and II. Springer-Verlag, New York.

Faraut, J. and A. Korányi (1994), Analysis on Symmetric Cones. Oxford University Press, New York.

Faybusovich, L. (1997), Euclidean Jordan algebras and interior-point algorithms, Positivity 1, 331-357.

Fukushima, M. (1992), Equivalent differentiable optimization problems and descent methods for asymmetric variational inequality problems, Mathematical Programming 53, 99-110.

Fukushima, M. (1996), Merit functions for variational inequality and complementarity problems. In: Di Pillo G., Giannessi F. (eds.) Nonlinear Optimization and Applications, Plenum Press, New York, 155-170.

Gowda, M.S., R. Sznajder and J. Tao (2004), Some P-properties for linear transformations on Euclidean Jordan algebras, Linear Algebra and Its Applications 393, 203-232.

Gowda, M.S. and R. Sznajder (2006), Automorphism invariance of P and GUS properties of 
linear transformations on Euclidean Jordan algebras, Mathematics of Operations Research 31, 109-123.

Gowda, M.S. and J. Tao (2007), Z-transformation on proper and symmetric cone, Mathematical Programming, Published online: 18 July 2007.

Isac, G. (2000), Topological Methods in Complementarity Theory. Kluwer Academic Publishers, Dordrecht.

Kanzow, C. and M. Fukushima (1998), Theoretical and numerical investigation of the D-gap function for box constrained variational inequalities, Mathematical Programming 83, 55-87.

Kanzow, C. and M. Fukushima (1998), Solving box constrained variational inequalities by using the natural residual with D-gap function globalization, Operations Research Letters 23, 45-51.

Koecher, M (1999), The Minnesota Notes on Jordan Algebras and Their Applications, edited and annotated by Brieg, A., Walcher, S., Springer, Berlin.

Kong, L.C. and N.H. Xiu (2007), New smooth C-functions for symmetric cone complementarity problems, Optimization Letters 1, 391-400.

Lin, Y. and A. Yoshise (2005), A homogeneous model for mixed complementarity problems over symmetric cones, Preprint, University of Tsukuba, Japan.

Liu, Y., L. Zhang and Y. Wang (2006), Some properties of a class of merit functions for symmetric cone complementarity problems, Asia-Pacific Journal of Operational Research 23, 473-495.

Luo, Z.Q., O.L. Mangasarian, J. Ren and M.V. Solodov (1994), New error bounds for the linear complementarity problems, Mathematics of Operations Research 19, 880-892.

Luo, Z.Q., J.-S. Pang and D. Ralph (1996), Mathematical Programms with Equilibrium Constraints, Cambridge University Press.

Malik, M. and S.R. Mohan (2003), On complementarity problems over symmetric cones, Discussion Paper Series DPS/SQCOR/Delhi/05-2003, Indian Statistical Institute.

Malik, M. and S.R. Mohan (2006), Cone complementarity problems with finite solution sets, Operations Research Letters 34, 121-126.

Mangasarian, O.L. and M.V. Solodov (1993), Nonlinear complementarity as unconstrained and constrained minimization, Mathematical Programming 62, 277-297.

Peng, J.M. (1997), Equivalence of variational inequality problems to unconstrained minimization, Mathematical Programming 78, 347-355.

Peng, J.M. (1997), Convexity of the Implicit Lagrangian, Journal of Optimization Theory and Applications 92, 331-341. 
Peng, J.M. and M. Fukushima (1999), A hybrid Newton method for solving the variational inequality problem via the D-gap function, Mathematical Programming 86, 367-386.

Rockafellar, R.T. and R.J.-B. Wets (2004), Variational Analysis, Second Version. Springer, New York.

Schmieta S.H. and F. Alizadeh (2003), Extension of primal-dual interior point algorithms to symmetric cones, Mathematical Programming 96, 409-438.

Solodov, M.V. and P. Tseng (2000), Some methods based on the D-gap function for solving monotone variational inequalities, Computational Optimization and Applications 17, 255-277.

Sun, D., M. Fukushima and L. Qi (1997), A computable generalized Hessian of the D-gap function and Newton-type methods for variational inequality problems. In: Ferris, M.C., Pang, J.-S. (Eds.), Complementarity and Variational Problems: State of the Art, SIAM, Philadelphia, PA, 452-473.

Sun, D. and L. Qi (1999), On NCP-functions, Computational Optimization and Applications $13,201-220$.

Sun, D. and J. Sun (2008), Löwner's operator and spectral functions on Euclidean Jordan algebras, Mathematics of Operations Research 33.

Tao, J. and M.S. Gowda (2005), Some P-properties for nonlinear transformations on Euclidean Jordan algebras, Mathematics of Operations Research 30, 985-1004.

Tseng, P. (1998), Merit function for semi-definite complementarity problems, Mathematical Programming $83,159-185$.

Tseng, P., N. Yamashita and M. Fukushima (1996), Equivalence of complementarity problems to differentiable minimization: A unified approach, SIAM Journal on Optimization 6, 446-460.

Yamashita, N. and M. Fukushima (1995), On stationary points of the Implicit Lagrangian for nonlinear complementarity problems, Journal of Optimization Theory and Applications 84, 653663.

Yamashita, N., K. Taji and M. Fukushima (1997), Unconstrained optimization reformulations of variational inequality problems, Journal of Optimization Theory and Applications 92, 439-456.

Yoshise, A. (2006), Interior point trajectories and a homogeneous model for nonlinear complementarity problems over symmetric cones, SIAM Journal on Optimization 17, 1129-1153.

Kong Lingchen is a Post-Doctoral Fellow of Department of Combinatorics and Optimization, Faculty of Mathematics, University of Waterloo, Canada. He received his MS degree in Management from Shandong University of Science and Technology in 2004 and PhD degree in Operations Research from Beijing Jiaotong University in 2007. His research interests are in mathematical optimization, mathematics of operations research. 
Tunçel Levent is a Professor in Department of Combinatorics and Optimization, Faculty of Mathematics, University of Waterloo, Canada. He received his $\mathrm{PhD}$ degree from Cornell University in 1992. His research interests are mostly in mathematical optimization, mathematics of operations research and foundations of computational mathematics. Research of this author was supported in part by a Discovery Grant from NSERC.

Xiu Naihua is a Professor in Department of Applied Mathematics, Beijing Jiaotong University. He received his PhD degree in Operations Research from Academy of Mathematics and System Sciences of the Chinese Academy of Sciences in 1997. He was a Research Fellow of City University of Hong Kong from 2000 to 2002, and he was a Visiting Scholar of University of Waterloo from 2006 to 2007. His research interest includes variational analysis, mathematical optimization and mathematics of operations research. 